\title{
Providing maternal and newborn health services: Experiences of auxiliary nurse midwives in Rajasthan
}

\author{
K.G. Santhya \\ Population Council \\ Shireen J. Jejeebhoy \\ Population Council
}

Follow this and additional works at: https://knowledgecommons.popcouncil.org/departments_sbsr-pgy

Part of the Demography, Population, and Ecology Commons, Family, Life Course, and Society Commons, International Public Health Commons, Maternal and Child Health Commons, and the Maternal, Child Health and Neonatal Nursing Commons

How does access to this work benefit you? Let us know!

\section{Recommended Citation}

Santhya, K.G. and Shireen J. Jejeebhoy. 2012. "Providing maternal and newborn health services:

Experiences of auxiliary nurse midwives in Rajasthan." New Delhi: Population Council. 


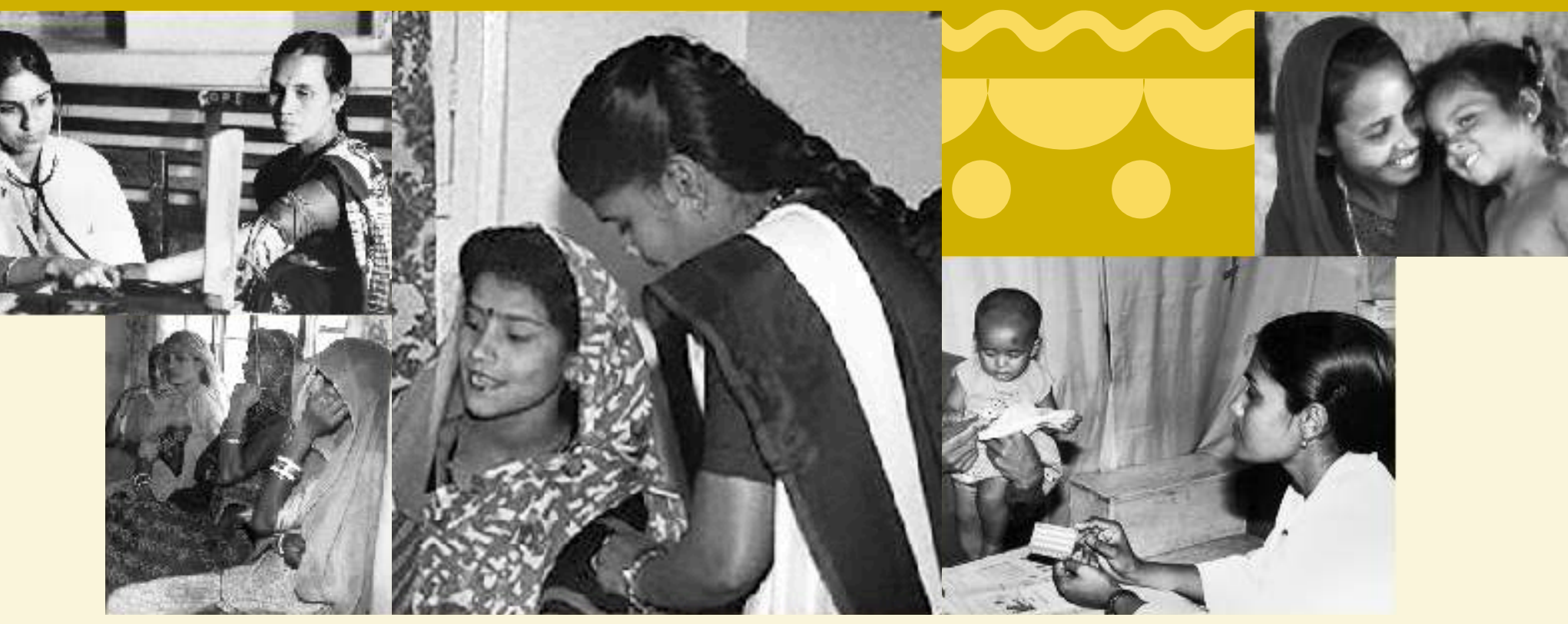

\section{Providing maternal and newborn health services: Experiences of Auxiliary Nurse Midwives in Rajasthan}


This report is the result of an exploratory study of Auxiliary Nurse Midwives (ANMs), conducted as part of a large-scale evaluation of the Janani Suraksha Yojana in rural and urban settings of the north-western state of Rajasthan, India. The study assessed the experiences of ANMs in providing maternal and newborn health services and their perspectives on the Janani Suraksha Yojana.

For additional copies of this report, please contact:

Population Council

Zone 5-A, Ground Floor

India Habitat Centre

Lodi Road

New Delhi -110003

Phone: 011-2464 2901/02

Email: info-india@popcouncil.org

Web site: http://www.popcouncil.org/asia/india/html

The Population Council is an international, non-profit, non-governmental organisation that seeks to improve the well-being and reproductive health of current and future generations around the world and to help achieve a humane, equitable and sustainable balance between people and resources. The Council conducts biomedical, social science and public health research, and helps build research capacities in developing countries.

\section{Copyright $(2011$ Population Council}

Suggested citation: Santhya, K. G and S. J. Jejeebhoy. 2012. Providing maternal and newborn health services: Experiences of Auxiliary Nurse Midwives in Rajasthan New Delhi: Population Council. 


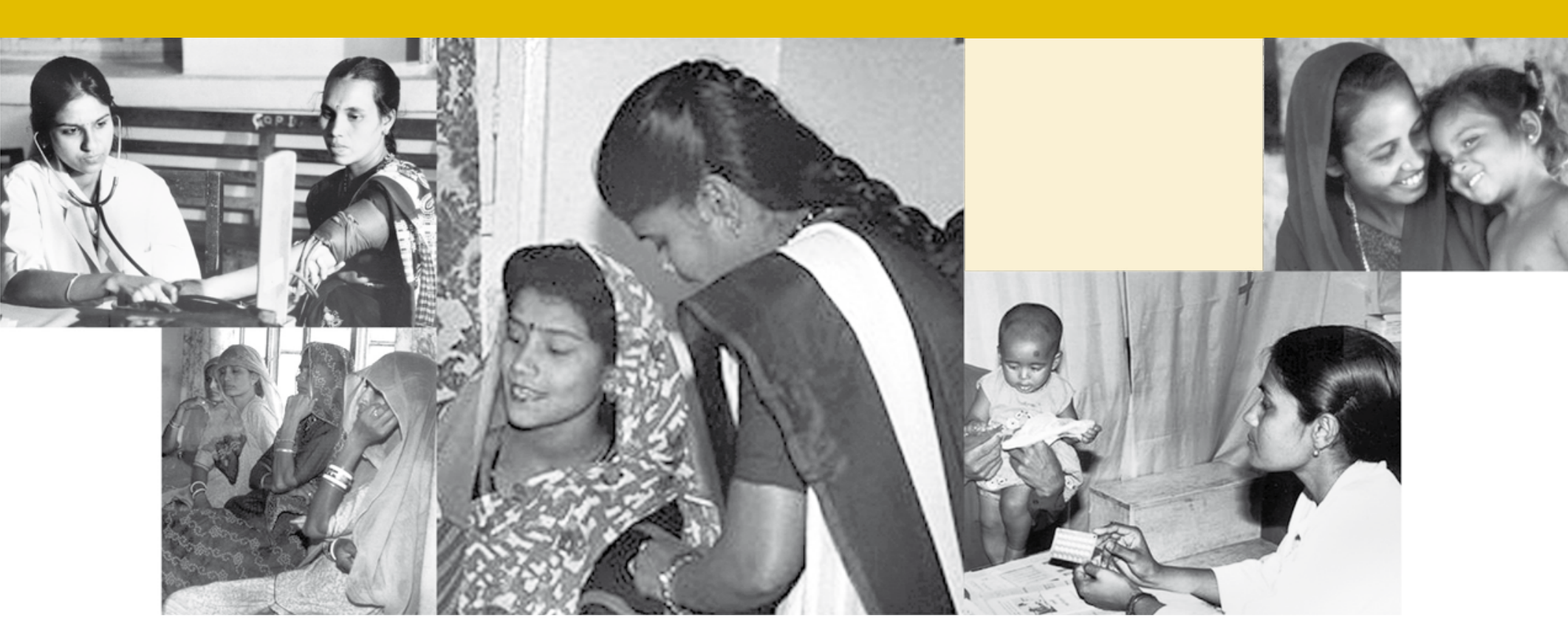

\section{Providing maternal and newborn health services: Experiences of Auxiliary Nurse Midwives in Rajasthan}

K. G. Santhya

Shireen J. Jejeebhoy 



\section{Contents}

Acknowledgements

vii

Executive summary

viii

Recommendations

Chapter 1: Introduction

Background

Study objectives

Study setting

Study design

Characteristics of respondents

Characteristics of the facility in which ANMs were working

Structure of the report

Chapter 2: Extent of training received by ANMs

$\begin{array}{ll}\text { Training received on imparting information on sexual and reproductive matters } & 7\end{array}$

$\begin{array}{lc}\text { Special training received for providing maternal health services } & 8\end{array}$

Chapter 3: Awareness of maternal and newborn care practices among ANMs 9

Awareness of antenatal care practices $\quad 9$

Awareness of birth preparedness and selected protocols to be followed during labour and delivery $\quad 10$

$\begin{array}{ll}\text { Awareness of postpartum care practices } & 11\end{array}$

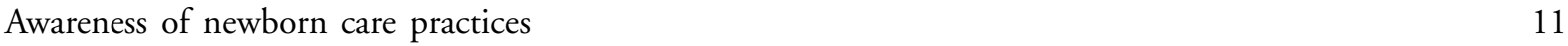

Awareness of complications during pregnancy, delivery and the postpartum period,

and among newborns 13

$\begin{array}{lr}\text { Chapter 4: Services provided by ANMs } & 18\end{array}$

$\begin{array}{ll}\text { Antenatal care-related services } & 18\end{array}$

$\begin{array}{ll}\text { Delivery care-related services } & 21\end{array}$

$\begin{array}{ll}\text { Postpartum and newborn care-related services } & 23\end{array}$ 
Chapter 5: ANM's awareness of and perspectives on the JSY

Awareness of the eligibility conditions for availing of the JSY

Awareness of women's and ASHAs' entitlements under the JSY

ANMs' awareness of schemes other than the JSY

ANMs' perceptions about the JSY

Chapter 6: Summary and recommendations

Summary

Recommendations

References

Members of the study team

Authors 


\section{List of tables}

Table $1.1 \quad$ Profile of the study districts and state 4

Table $1.2 \quad$ Socio-demographic characteristics of respondents 5

Table 1.3 Characteristics of the facility in which the respondents were working 6

Table 2.1 Extent to which ANMs received training on imparting information on sexual and reproductive matters 7

Table 2.2 Extent to which IEC materials were available to ANMs on sexual and reproductive topics $\quad 7$

Table 2.3 Special training received for providing maternal health services $\quad 8$

Table $3.1 \quad$ Awareness of antenatal care practices 9

Table 3.2 Awareness of birth preparedness and selected protocols to be followed $\begin{array}{ll}\text { during labour and delivery } & 10\end{array}$

Table 3.3 Awareness of newborn care practices 12

Table 3.4 Awareness of complications during pregnancy, delivery and the postpartum period, and among newborns 14

Table 3.5 ANMs' awareness of recommended facilities to which to refer a pregnant woman who experiences selected pregnancy-related complications 16

Table 3.6 Awareness of proper management of selected pregnancy-related complications 16

Table 4.1 Percentages of ANMs reporting that they had followed standard protocols while conducting antenatal check-ups in the three months preceding the interview

Table 4.2 Percentage of ANMs reporting that they had followed standard protocols of delivery care and abstained from harmful practices

Table 4.3 Percentages of ANMs reporting that they had provided postpartum care-related services in the three months preceding the interview

Table 4.4 Percentage of ANMs reporting that they had followed standard protocols of newborn care in deliveries conducted in the three months preceding the interview 24

Table 5.1 Awareness of eligibility conditions for availing of the JSY 27

Table 5.2 Awareness of women's monetary and non-monetary entitlements under the JSY 27

Table 5.3 Awareness of ASHA's entitlements under the JSY 28

Table 5.4 Percentage of ANMs reporting changes in job responsibilities and utilization of maternal health services with the introduction of the JSY 


\section{List of figures}

Figure 2.1 Percentage of ANMs reporting availability and use of IEC materials on sexual and reproductive topics

Figure 3.1 Percentage of ANMs who were aware of the protocols to be followed during labour and delivery

Figure $3.2 \quad$ Awareness of postpartum care practices

Figure 3.3 Percentage of ANMs who spontaneously mentioned the complications during pregnancy, delivery and postpartum period

Figure 4.1 Percentages of ANMs reporting that they maintained privacy for women and had good interactions with women

Figure 4.2 Delivery care-related services provided by ANMs 21

Figure 5.1 Awareness of schemes other than the JSY 


\section{Acknowledgements}

This study has benefited immeasurably from the input of many. We are extremely grateful to the John D. and Catherine T. MacArthur Foundation for their support, which made this study possible.

The Department of Health and Family Welfare, Rajasthan granted permission to conduct the study and offered their unstinting support at all stages of the project and we take this opportunity to express our gratitude for their support and guidance to Meghraj Meena, Former District Reproductive and Child Health Officer, Alwar; Nanda Charan, Reproductive and Child Health Officer, Jodhpur; Subodh Agarwal and Ramesh Mathur, Former Chief Medical and Health Officers, Alwar and Jodhpur districts respectively; and Mohit Sharma, Former District Programme Manager, Jodhpur. Our thanks also go to the grass-root level workers of the department who generously supported the field team in data collection. The State Census Office in Rajasthan provided the project with census enumeration block maps and their support is gratefully acknowledged.

The Technical Advisory Group—Dinesh Agarwal, Kiran Ambwani, Dipa Nag Chowdhury, Aparajita Gogoi, Poonam Muttreja, Saroj Pachauri, Amit Mohan Prasad and Rajni Ved—provided invaluable advice in developing the study instruments, project design, and interpreting the findings.

We acknowledge with gratitude the contribution of Kurus Coyaji and Dinesh Agarwal for reviewing an earlier draft of this report and providing insightful comments and suggestions. We would like to gratefully acknowledge support received from Rajib Acharya for developing the study instruments and the data entry package that enabled interviewers to conduct interviews and record responses directly onto mini laptops, Shveta Kalyanwala for developing the study instruments and training the field team, A. J. Francis Zavier for analysing the data and (Late) Rajesh Kumar for translating the instruments. The contributions of Shanti Menon in editing the report, Komal Saxena in preparing the report and ably coordinating the printing of the report, Shilpi Rampal for testing the data entry package, M. A. Jose for ably managing the administrative aspects of this project and B. Srihari for the overall management of the field work are much appreciated.

Finally, and most importantly, we would like to thank the Auxiliary-Nurse-Midwives of Alwar and Jodhpur districts, Rajasthan who generously gave us their time and shared their views and experiences, and the investigators and other members of the field team who painstakingly collected the qualitative and survey data.

K. G. Santhya

Shireen J. Jejeebhoy 


\section{Executive summary}

Several measures have been taken in recent years under the National Rural Health Mission to improve the competence of Auxiliary Nurse Midwives (ANMs) to provide maternal and newborn health services. Guidelines have been established for the provision of antenatal care services and skilled attendance at birth by ANMs and Lady Health Visitors (LHVs); a training programme to upgrade the skills of ANMs and staff nurses to improve skilled attendance at birth has been introduced; and staff nurses and ANMs are currently allowed to initiate treatment of pregnancy-related complications. Studies exploring the perspectives and experiences of ANMs in delivering maternal and newborn health services following the introduction of the NRHM are, however, limited. To better understand the experiences of ANMs in providing maternal and newborn health services post NRHM and to obtain their perspectives on the Janani Suraksha Yojana (JSY), the Population Council undertook a study of ANMs in two districts of the north-western state of Rajasthan, India.

The study was conducted as part of a large-scale evaluation of the JSY, conducted between September 2009 and February 2010, that comprised a survey of nearly 5,000 women aged below 35 years who had delivered in the one year preceding the interview and in-depth interviews with selected survey respondents. The study team visited sub-centres, primary health centres, government dispensaries and community health centres located within the villages and urban wards selected for the survey of women in order to enroll ANMs in the study. ANMs available in these facilities were invited to participate in the study. A total of 125 ANMs were listed. Of these, 96 ANMs were successfully interviewed, 24 from urban areas and 72 from rural areas.

\section{The majority of ANMs were trained in delivering maternal and newborn health services}

Almost all the ANMs had received training in imparting information pertaining to contraception, care during pregnancy, delivery and the postpartum period, and newborn care. Moreover, the vast majority of ANMs (75-93\%) had received training in conducting such tasks as measuring blood pressure, checking pallor, measuring fundal height, determining foetal presentation, conducting a normal delivery, giving uterotonic drugs to enhance contraction of uterine muscles, carrying out controlled cord traction and carrying out uterine massage; a little over half of the ANMs had received training in conducting all of these tasks.

\section{Awareness of maternal and newborn care practices is not comprehensive}

Awareness of best maternal and newborn care practices was, for the most part, high among the ANMs. For example, awareness of the number of antenatal check-ups, the appropriate timing of the first antenatal check-up, number of doses of tetanus toxoid injection, appropriate timing for receiving the first dose of TT injection and number of days that iron and folic acid supplements should be taken was almost universal. However, awareness of the appropriate timing of the second and third check-ups and the number of days that a severely anaemic woman should take iron and folic supplements was somewhat limited. 
The majority of the ANMs were aware of some of the essential birth preparations; however, only $44 \%$ were able to spontaneously mention three or more preparations for a safe delivery. Awareness of standard protocols to be followed during labour and delivery was limited; only 30\% of ANMs knew that it is not advisable to conduct a vaginal examination if the woman is bleeding at the time of labour or to give oxytocin to speed up delivery, and that using oxytocin or misoprostol for active management of the third stage of labour is recommended.

Awareness of postpartum care practices among the ANMs was far from universal. Only four-fifths of the ANMs knew that a woman should have her first check-up within a few hours or at best within two days of delivery. Only half of the ANMs knew that a woman should have three postpartum check-ups.

Findings show that the vast majority of the ANMs were aware of best practices related to the immediate care of the newborn. Awareness of appropriate feeding practices and the recommended immunization schedule, likewise, was high.

The majority of the ANMs were aware of complications during pregnancy and complications among the newborns; three-quarters were able to list at least three severe complications during pregnancy and a little over half were able to list at least three severe complications among newborns. However, fewer were aware of complications during labour and delivery and the postpartum period; just 32\% and 19\% were able to list at least three severe complications during labour and delivery, and the postpartum period. Findings, moreover, indicate limited awareness of appropriate health facilities for treating pregnancy-related complications. For example, just one-third of the ANMs knew that a pregnant woman who experiences puffiness of the face must go to a primary health centre and a woman who experiences labour lasting more than 12 hours should go to a first referral unit, that is, a community health centre.

Finally, the ANMs' awareness of proper management of selected complications was limited. For example, only $16 \%$ of ANMs knew that if a pregnant woman who experiences pre-eclampsia or whose delivery is imminent approaches the ANM, she should conduct the delivery rather than refer the patient to an FRU without conducting the delivery.

\section{Standard protocols of care are not often followed while delivering maternal and newborn care-related services}

All the ANMs who participated in the study reported that they had provided maternal and newborn care-related services in the three months preceding the interview. Notably, some 37\% of ANMs had conducted a delivery in the three months preceding the interview. However, standard protocols of care were not often followed while delivering these services and the services that were extended were far from comprehensive.

In the area of antenatal care services, findings show that while almost all the ANMs reported that they took a medical history whenever a pregnant woman came for her first antenatal check-up, it was largely limited to enquiring about the woman's previous pregnancy history. Likewise, although over $90 \%$ of ANMs reported that they conducted physical examinations as part of antenatal check-ups, these comprised just measuring weight and, to some extent, conducting an abdominal examination and checking blood pressure. 
No more than half of the ANMs reported that they had conducted basic laboratory investigations. While almost all the ANMs reported that they spent some 10-15 minutes in consultation and that they always asked the woman whether she had any questions to discuss with them, auditory privacy was ensured by just a few and even visual privacy was not ensured by all.

With regard to delivery care, findings show that most of the ANMs who had conducted a delivery in the three months prior to the interview reported that they followed several of the standard protocols - conducting all the essential examinations whenever a woman was admitted to the facility for delivery, using clean gloves while conducting vaginal examinations and washing hands before and after conducting vaginal examinations. However, just a few ANMs reported following such protocols as conducting vaginal examinations about once every four hours and not more often, using the partograph, and using oxytocin or misoprostol for the active management of the third stage of labour. Findings also indicate that such harmful practices as applying strong fundal pressure before the woman delivered and using intra-muscular injection to speed up delivery were common, particularly the latter. All the ANMs reported that they ensured visual privacy for the women during delivery and the majority reported that they discharged the women only after at least 24 hours of delivery. However, most ANMs reported that they asked the women or their family members to arrange medicine and other supplies, and few reported that they allowed the person accompanying the women to remain with them during delivery.

Almost all the ANMs reported that they had visited recently delivered women in the last three months, although not many made the first postpartum visit within 2 days of delivery. The majority of the ANMs reported that they usually counseled recently delivered women about self-care, care of the newborn, breastfeeding and contraception. Finally, almost all the ANMs reported that they had followed best practices related to newborn care, for example, never delivering the newborn on to the floor, cutting the cord after 2-3 minutes of delivery, not applying any substance on the cord stump, always wiping the newborn dry and wrapping immediately instead of bathing her/him, encouraging the mother to breastfeed the newborn immediately, and encouraging the mother to feed colostrum to the newborn.

\section{Awareness of the JSY among ANMs is far from comprehensive}

Awareness of the JSY programme among ANMs was far from comprehensive. The vast majority of the ANMs were aware of most of the eligibility conditions for availing of JSY benefits, including the fact that women could access JSY benefits regardless of their age, parity and household economic status, as well as the type of government health facility in which they could deliver to obtain these benefits. However, their awareness of the monetary and non-monetary support to which women are entitled under the JSY was somewhat limited. For example, only 81 percent of the ANMs knew that a woman in a rural area is entitled to Rs. 1,400 and that a woman in an urban area is entitled to Rs. 1,000 if she delivers in a government or accredited private facility. Findings, moreover, indicate considerable confusion among the ANMs with regard to the entitlements of ASHAs who assist women in accessing JSY benefits. 


\section{Recommendations}

The findings of the study suggest a number of priority areas for action.

Increase investments in training programmes for ANMs and raise awareness of the lesser known best practices related to maternal and newborn care and complications during delivery and the postpartum period and management of these complications

Findings that not all ANMs had received the full training in providing maternal and newborn health services call for increased investments in training programmes for ANMs. Findings call for further efforts to raise ANMs' awareness of maternal and newborn care practices. These efforts must pay special attention to increasing their awareness of the complications that a woman may experience during labour and delivery, and the postpartum period, and the appropriate health facilities for treating pregnancy-related complications. Such efforts must also impart information to the ANMs about how to manage complications that ANMs are expected to take care of.

\section{Encourage ANMs to provide delivery and post partum care-related services on a more regular basis}

Our findings indicate that antenatal care-related services were provided by almost all the ANMs. However, just one third had provided delivery care, and very few had made postpartum visits to recently delivered women within two days of delivery. These findings call for emphasising the role of ANMs in providing delivery and postpartum care-related services.

\section{Make special efforts to enable ANMs to follow standard protocols of care}

Findings emphasise the need for special efforts to enable ANMs to follow standard protocols of care. Specifically, ANMs need to be oriented about the importance, while taking a woman's medical history, of enquiring about the date of the last menstrual period, any history of systemic illnesses, and symptoms experienced during pregnancy, as well as checking blood pressure, pallor and height while conducting antenatal check-ups. Likewise, in the area of delivery care, ANMs need to be trained and encouraged to use the partograph for monitoring labour and to use oxytocin or misoprostol for the active management of the third stage of labour. At the same time, they must be dissuaded from such harmful practices as applying fundal pressure before the woman delivers and using intra-muscular injections to speed up delivery. In the area of postpartum care, our findings call for orienting ANMs about the importance of making the first postpartum visit to recently delivered women within two days of delivery. Enabling ANMs to follow these standard protocols of care may require training programmes to upgrade their skills. The training must emphasise the need to provide good quality services as much as it focuses on developing technical skills. Also required are monitoring mechanisms to ensure that ANMs do follow the protocols. 
We also note that improvements in infrastructure at the health facility are required to enable ANMs to follow standard protocols of care, for example, to ensure privacy for the women or keep the delivered women at the facility for more than a day.

\section{Raise ANMs' awareness about the JSY programme and address weaknesses in the programme identified by ANMs}

ANMs recognised that the JSY had resulted in an increased demand for maternal health services. However, they were not fully aware of women's entitlements; efforts are needed to provide comprehensive information to ANMs about the JSY programme and its entitlements. At the same time, efforts must be made to address issues raised by ANMs about weaknesses in the implementation of the programme, for example, about inadequate staff at the PHC, lack of facilities for conducting deliveries at the sub-centre and JSY payment linked only to institutional delivery rather than a continuum of care, including immunization of infants. 


\section{CHAPTER 1}

\section{Introduction}

Significant measures have been taken, under the National Rural Health Mission (NRHM), to improve women's access to maternal and child health services, including, for example, schemes such as Janani Suraksha Yojana and Accredited Social Health Activists (ASHAs). Similarly, significant investments have been made in improving the health infrastructure and in strengthening provider capacity. For example, manuals have been developed that address different cadres of providers (ASHAs, Auxiliary Nurse Midwives (ANMs), Medical Officers (MOs)) and different thematic areas, including maternal and newborn health, and child health and nutrition. Efforts have also been made for multi-skilling of providers. Studies exploring the perspectives of providers on their experience in delivering maternal and newborn health services following the introduction of the NRHM are few.

Drawing on data from 96 ANMs interviewed as part of a large-scale evaluation of the JSY in two districts of Rajasthan, this report seeks to take stock of their awareness of maternal and newborn care practices and their experiences in the provision of maternal and newborn health services. It also presents evidence on their perspectives on the JSY. Corresponding insights from ASHAs are presented elsewhere (Santhya et al., 2011).

\section{Background}

In India, the most recent estimates (March 2010) suggest that there are about 191,457 ANMs. This represents an increase of 44\% since 2005 (Ministry of Health and Family Welfare, 2011). The current shortfall in the post of ANMs is $9 \%$ of the total requirement as per the norm of one ANM (or female health worker) per sub-centre and PHC, and $6 \%$ of sanctioned posts of ANMs are vacant.

The cadre of ANMs was established in the 1960s to provide maternal and child health services. However, their roles and responsibilities have changed over the decades with the shift in programme priorities nationally. As per the Indian Public Health Standards guidelines, the job responsibilities of ANMs encompass several thematic areas-maternal and child health services, family planning services, abortion-related referral, nutritional services for pregnant women and children, immunisation of pregnant women and children, training traditional birth attendants in promoting family planning, identification and notification of communicable diseases, treatment of minor ailments, record keeping, including of vital events, and provision of supportive supervision to ASHAs (Ministry of Health and Family Welfare, 2006a). Specifically related to maternal and child health services, the responsibilities of ANMs include (1) registering and providing care to pregnant women throughout the period of pregnancy, including antenatal check-ups and associated services such as the provision of IFA tablets and TT immunization; (2) conducting basic laboratory investigations, namely, testing the urine of pregnant women for albumin and sugar, checking haemoglobin levels, and referring pregnant women to the PHC for syphilis testing; (3) referring cases of abnormal or complicated pregnancy to PHCs; (4) conducting deliveries in their area when called 
for and supervising deliveries conducted by dais; (5) referring cases of difficult labour and newborns with abnormalities and helping them get institutional care; (6) making at least two postpartum visits for each delivery that took place in their area and counselling women about self-care and care of the newborn; (7) assessing the growth and development of the infant and taking necessary action to rectify the deficiencies, if any; and (8) providing supportive supervision of ASHAs and facilitating the implementation of the JSY.

Several researchers have commented that the tasks of ANMs are disproportionately focused on family planning and immunization services and such tasks as providing delivery and basic curative services are considerably neglected, which can adversely affect the maternal health situation in the country (Mavalankar and Vora, 2008; Mavalankar, Vora and Prakasamma, 2008, Vora et al., 2009). It has also been observed that efforts to train ANMs in essential obstetric functions, which could serve to stabilise women before referral to FRUs or prevent deaths due to complications, are limited (Ved and Dua, 2005). However, several measures have been taken in recent years under the NRHM to improve the competence of ANMs to provide maternal and newborn health services. For example, guidelines have been established for the provision of antenatal care services and skilled attendance at birth by ANMs and lady health visitors (LHVs) (Ministry of Health and Family Welfare, 2005; 2010). A training programme to upgrade the skills of ANMs and staff nurses to improve skilled attendance at birth has been introduced (Ministry of Health and Family Welfare, 2006b). Staff nurses and ANMs are currently allowed to initiate treatment of pregnancyrelated complications, including the administration of intravenous fluids and injectable oxytocics, antibiotics, and magnesium sulphate. Finally, provision for an additional ANM in the sub-centres has been made.

Studies exploring the perspectives and experiences of ANMs in delivering maternal and newborn health services following the introduction of the NRHM are limited. Indeed, most of the studies that have examined the perspectives of ANMs on the quality of services provided by them and the barriers they face in providing quality services were conducted in the 80s and 90s (Bhatia, 1999; Iyer and Jesani, 1999; Khan, Patel and Gupta, 1999; Verma and Roy, 1999; Visaria, 1999). Most of these studies have focussed on the delivery of family planning services. The few that have been conducted following the introduction of the NRHM suggest limited awareness of maternal care practices and standard protocols among ANMs. For example, a study of some 136 ANMs in two districts in Jharkhand observes that $13 \%$ were aware of partographs, $7 \%$ identified blurred vision as a complication during the antenatal period, and $15 \%$ and $25 \%$ recognised foetal distress and retained placenta, respectively, as complications during delivery (IntraHealth, 2008). It also reports that the majority of the ANMs were lacking in skills required to provide effective maternal health services; for example, just twofifths of the ANMs felt that they could handle such complications as night blindness and swelling around the ankles. A five-state study of nurses, including ANMs, reports that very few ANMs had used partographs or had received written protocols for managing maternal complications (Academy for Nursing Studies and Women's Empowerment Research Studies and National Health System Resource Centre, 2010a; 2010b; 2010c; 2010d; 2010e). Thus, there is a need to better understand, from the perspectives of ANMs, their competence in delivering maternal and newborn health services and the extent to which they have been following 
standard protocols of care while delivering these services.

\section{Study objectives}

The aim of this study is to better understand the experiences of ANMs in providing maternal and newborn health services post NRHM and to get their perspectives on the JSY. Specifically, the objectives of the study are to explore:

- The awareness of maternal and newborn care practices among ANMs

- Maternal and newborn care services provided by ANMs

- The perceptions of ANMs about the JSY

\section{Study setting}

The study was conducted in the state of Rajasthan. With a population of 68.6 million in 2011, the state ranks 8th in terms of the total population among states in India (Office of the Registrar General and Census Commissioner, India, 2011). With a child sex ratio of 883 females per 1,000 males in 2011, the state registered one of the most skewed sex ratios in the country. Rajasthan lags behind the rest of India in terms of social and health indicators as well. The state's overall literacy rate was $67 \%$ in 2011 compared to $74 \%$ nationally (Office of the Registrar General and Census Commissioner, India, 2011). With 65 percent of 20-24 year-old women married before age 18, the state ranks second among all states in India in terms of the prevalence of early marriage (International Institute for Population Sciences and Macro International, 2008). Moreover, childbearing in adolescence is widely prevalent in the state; two-fifths of currently married girls aged 15-19 years were already mothers and another one-tenth were pregnant at the time of the survey
(International Institute for Population Sciences and Macro International, 2008). With a maternal mortality ratio of 318 per 100,000 births, the state records the third highest maternal mortality ratio among all the states in India (Office of the Registrar General, India, 2011). The utilisation of maternal health services is limited (see Table 1). A recent assessment, conducted in 2009-10, also reaffirms that the utilisation of maternal health services is far from satisfactory-only 55\% of women had three or more antenatal check-ups and 70\% had delivered in a health facility (United Nations Children's Fund, 2010). The coverage of centrallysponsored programmes to promote maternal health, like the Janani Suraksha Yojana, remains far from universal. For example, an evaluation conducted by the UNFPA reports that just one-half of women who had delivered in the one year preceding the survey had received financial assistance under the JSY (United Nations Population Fund, 2009).

With regard to the availability of ANMs, the state recorded a surplus as on March 2010; the state has 16,086 ANMs compared to 12,991 ANMs required (Ministry of Health and Family Welfare, 2011).

Two districts, Alwar and Jodhpur, were purposively selected for the study. These districts represent the state averages in selected sociodemographic and reproductive health indicators such as male and female literacy rates, percentages of girls married below the legal minimum age at marriage, percentages of women who were currently using contraception and percentages of women who had skilled attendance at delivery. They also represent the eastern and western regions of the state. A few key indicators of the study districts are presented in Table 1.1. Data were collected in both urban and rural areas of these two districts. The selection of villages in rural areas and census enumeration blocks in the urban area within each district was 
Table 1.1

Profile of the study districts and state

\begin{tabular}{|c|c|c|c|}
\hline Characteristics & Alwar District & Jodhpur District & Rajasthan State \\
\hline Population $^{1}$ & $3,671,999$ & $3,685,681$ & $68,621,012$ \\
\hline Overall sex ratio $(\mathrm{F} / \mathrm{M})^{1}$ & 894 & 915 & 926 \\
\hline Child sex ratio $(0-6$ years $)(F / M)^{1}$ & 861 & 890 & 883 \\
\hline Male literacy $(\%)^{1}$ & 85.1 & 80.1 & 80.5 \\
\hline Female literacy $(\%)^{1}$ & 56.8 & 52.6 & 52.7 \\
\hline $\begin{array}{l}\text { Of those married in the last } 3 \text { years, } \\
\text { females married before age } 18(\%)^{2}\end{array}$ & 40.7 & 40.6 & 39.9 \\
\hline $\begin{array}{l}\text { Married women aged } 15-49 \text { currently using } \\
\text { any contraceptive method }(\%)^{2}\end{array}$ & 61.2 & 51.7 & 58.1 \\
\hline $\begin{array}{l}\text { Married women aged } 15-49 \text { who reported } \\
\text { skilled attendance at last delivery }(\%)^{2}\end{array}$ & 51.8 & 47.4 & 52.6 \\
\hline
\end{tabular}

done using a two-stage stratified systematic random sampling procedure. In the first stage of selection, three rural blocks and two or three urban blocks were selected. The blocks were first explicitly stratified by population size and the percentage of the population belonging to scheduled castes and tribes and then implicitly stratified by the level of female literacy. The blocks were then selected systematically from the stratified list, with selection probability proportional to size. Six blocks in rural areas and five blocks in urban areas were thus selected for the study. At the second stage, villages in rural areas and census enumeration blocks in urban areas were selected within each selected block, using a similar scheme. A total of 196 sampling units were thus selected.

\section{Study design}

The study was conducted between September 2009 and February 2010 as part of a large-scale evaluation of the JSY that comprised a survey of nearly 5,000 women aged below 35 years who had delivered in the one year preceding the survey, and in-depth interviews of selected survey respondents. The study team visited the sub-centres, primary health centres, government dispensaries and community health centres located within the villages and urban wards selected for the survey of women in order to enroll ANMs in the study. ANMs available in these facilities were invited to participate in the study.

A detailed questionnaire was administered to ANMs who consented to participate in the study. It was translated into the local language, Hindi, pre-tested and further modified. In addition to questions on background characteristics, the questionnaire included detailed questions about the ANM's awareness of maternal and newborn care practices, experiences in providing maternal and newborn care services, awareness of the JSY and perceptions about the JSY.

Interviewers were recruited locally. A ten-day training workshop was organised for the larger study to acquaint the interviewers with the questionnaire and interview guidelines and the method of conducting computer-assisted personal interviews. Data received from the field were regularly checked for quality, and feedback was provided to the investigators. 
A total of 125 ANMs were listed. Of these, 96 ANMs were successfully interviewed, 24 from urban areas and 72 from rural areas. While no ANM who was contacted refused to participate in the study, some $23 \%$ of the ANMs were not interviewed as they were not at the facility even after three visits. The survey data were analysed using SPSS. Given the small number of ANMs interviewed, particularly in urban areas, findings are presented for the combined sample in all the tables; however, insights pertaining to rural and urban samples are described in the text, as appropriate.

\section{Characteristics of respondents}

The socio-demographic characteristics of respondents are summarised in Table 1.2. On average, the respondents were aged 40 years, with urban respondents three years older than rural respondents (42 years versus 39 years). The majority of the respondents had completed 12 years of schooling, with no rural-urban differences. Respondents had been working as ANMs, on average, for 15 years, with little rural-urban difference (15 and 16 years respectively). They had been working, moreover, in the facility in which they were interviewed for about 7 years on average; ANMs in rural areas reported a longer period of service in the facility in which they were interviewed compared to their urban counterparts (8 years versus 3 years). Just $41 \%$ of ANMs were residing in the same village or urban ward in which the health facility in which they were working was located; rural-urban differences were narrow (10 out of 24 versus 29 out of 72 ).

\section{Characteristics of the facility in which ANMs were working}

Table 1.3 presents the characteristics of the facility in which the respondents were working at the time of the interview. Almost two-thirds of the ANMs who participated in the study were practising in a sub-centre, one-fifth in a primary health centre, one-tenth in a government dispensary and a small minority in a community health centre (4\%). Threequarters of the ANMs reported that the facility in which they were working was located in rural areas and the rest in peri-urban or urban areas. Almost all the ANMs (96-99\%) reported that the facility in which they were working provided such newborn and child care-related services as immunization of newborns and treatment of diarrhoea. Likewise, almost all the ANMs reported that antenatal and postpartum care services were provided in their facility (98-99\%). However, only half of the ANMs reported that delivery services were provided in their facility; this is not surprising given that $63 \%$ of ANMs were practising in a sub-centre. With regard to services related to the diagnosis and treatment of reproductive tract infections/sexually transmitted infections, while $93 \%$ of ANMs reported that services to diagnose RTIs/STIs were provided in

\section{Table 1.2}

\section{Socio-demographic characteristics of respondents}

\begin{tabular}{l|r|} 
Characteristics & N=96 \\
Mean age & 39.5 \\
Mean years of schooling & 11.6 \\
Mean years of working as an ANM & 15.1 \\
Mean years of working in the facility in which the respondent was interviewed & 6.7 \\
$\begin{array}{l}\text { Resides in the village/urban ward in which the health facility in which the } \\
\text { respondent was working is located (\%) }\end{array}$ & 40.6
\end{tabular}


Table 1.3

Characteristics of the facility in which the respondents were working

\begin{tabular}{|c|c|}
\hline Characteristics & $N=96$ \\
\hline \multicolumn{2}{|l|}{ Type of facility ${ }^{1}$} \\
\hline Sub-centre & 62.5 \\
\hline Primary health centre & 19.8 \\
\hline Government dispensary & 10.4 \\
\hline Community health centre & 4.2 \\
\hline \multicolumn{2}{|l|}{ Locality of the facility } \\
\hline Urban & 20.8 \\
\hline Peri-urban & 5.2 \\
\hline Rural & 74.0 \\
\hline \multicolumn{2}{|l|}{ Services available at the facility } \\
\hline Immunization & 99.0 \\
\hline Treatment for diarrhea & 95.8 \\
\hline Antenatal care services & 99.0 \\
\hline Delivery care services & 51.0 \\
\hline Postpartum care services & 97.9 \\
\hline Diagnosis of reproductive tract infections/sexually transmitted infections & 92.7 \\
\hline Treatment of reproductive tract infections/sexually transmitted infections & 59.4 \\
\hline Family planning counseling & 100.0 \\
\hline Provision of condoms & 100.0 \\
\hline Provision of oral contraceptives & 100.0 \\
\hline Insertion of Intrauterine Device & 85.4 \\
\hline
\end{tabular}

their facility (most likely syndromic management), $59 \%$ reported that treatment services were provided. Finally, almost all the ANMs reported that such contraceptive services as counselling and provision of condoms and oral contraceptives were provided in their facility, although somewhat fewer reported IUD insertion services. Rural-urban differences were evident with regard to the provision of delivery care services and insertion of IUD, with more ANMs in rural areas than in urban areas reporting that these services were provided in their health facility ( 42 out of 72 versus 7 out of 24 with regard to delivery care services; 67 out of 72 versus 15 out of 24 with regard to IUD insertion).

\section{Structure of the report}

The report is structured as follows. Chapter 2 describes the training received by ANMs in performing various tasks assigned to them, particularly, those related to maternal and newborn care. Chapter 3 examines the awareness of maternal and newborn care practices among ANMs. Chapter 4 describes the maternal and newborn care services extended by ANMs and the extent to which they have followed standard protocols of care. Chapter 5 describes ANMs' awareness of and perspectives on the JSY. Chapter 6 summarises the main findings of the study, and suggests programme recommendations. 


\section{CHAPTER 2}

\section{Extent of training received by ANMs}

The study included several question to assess the extent to which ANMs had been trained to provide maternal and newborn care-related services. Findings are presented in this chapter.

\section{Training received on imparting information on sexual and reproductive matters}

We assessed whether the ANMs had received training on imparting information on sexual and reproductive matters. Findings show that over 90\% of ANMs had received training on imparting information pertaining to contraception, care during pregnancy, delivery and the postpartum period, and newborn care (Table 2.1). Rural-urban differences were narrow.
ANMs were also asked whether IEC materials were available to them on sexual and reproductive topics and whether they had ever used them for imparting information on these topics. The majority (78-81\%) of ANMs reported that IEC materials were generally available on such topics as contraception, care during pregnancy, delivery and the postpartum period, and newborn care. Some $85 \%$ of ANMs reported availability of IEC materials on at least one topic. Rural-urban differences indicate that ANMs in rural areas were more likely than their urban counterparts to report the availability of IEC materials on all topics.

Almost three-quarters of ANMs reported that these materials were easily accessible; more rural than urban ANMs so reported (56 out of 72 versus

Table 2.1

Extent to which ANMs received training on imparting information on sexual and reproductive matters

\begin{tabular}{l|r} 
Topics on which training received & N=96 \\
\hline Contraception & 92.7 \\
Care during pregnancy & 94.8 \\
Care during delivery & 95.8 \\
Care during the postpartum period & 94.8 \\
Newborn care & 95.8
\end{tabular}

\section{Table 2.2}

Extent to which IEC materials were available to ANMs on sexual and reproductive topics

\section{Topics on which IEC materials were available}

Contraception

Care during pregnancy

Care during delivery

Care during the postpartum period

Newborn care

At least one of the above

\section{$\mathrm{N}=96$}

80.2

79.2

79.2

81.3

78.1

85.4 
Figure 2.1: Percentage of ANMs reporting availability and use of IEC materials on sexual and reproductive topics

Perceptions about and use of IEC materials $(\mathrm{N}=96)$

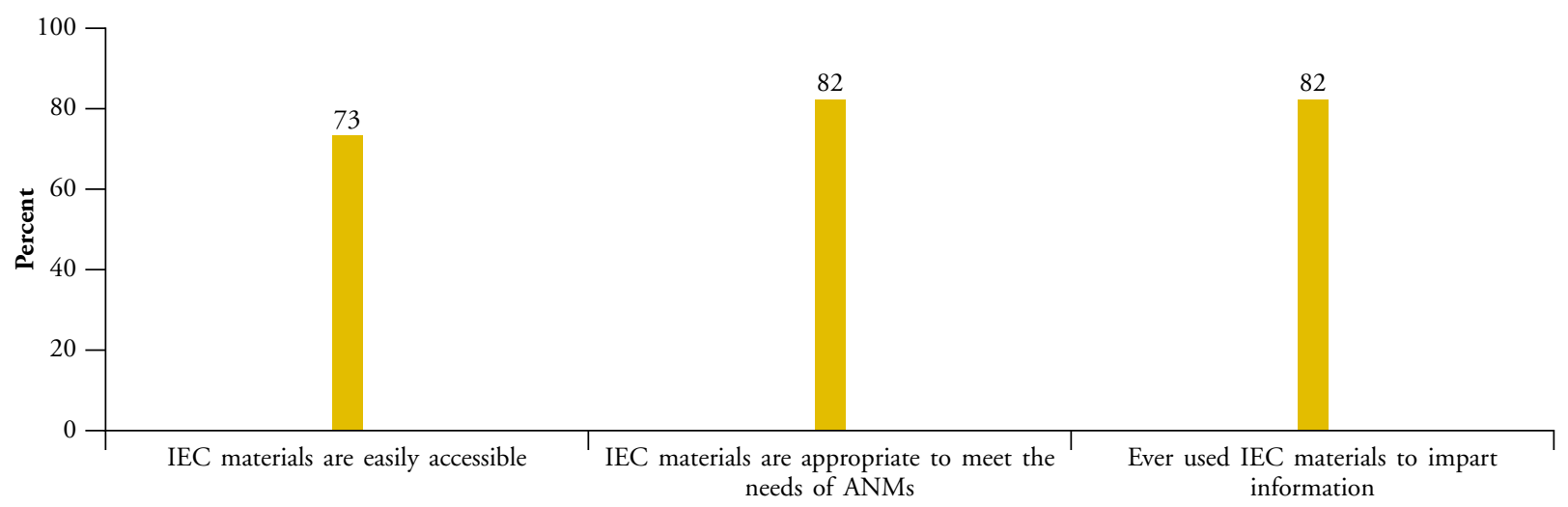

14 out of 24). Four-fifths of ANMs reported that the existing materials were appropriate for meeting the needs of health care providers like them and that they had used these materials in imparting information; again, rural ANMs were more likely than urban ANMs to so report (66 out of 72 versus 13 out of 24$)$.

\section{Special training received for providing maternal health services}

We also probed whether ANMs had received special training on providing maternal health services.
Specifically, we probed about training on measuring blood pressure, checking pallor, measuring fundal height, determining foetal presentation, conducting a normal delivery, giving uterotonic drugs to enhance the contraction of uterine muscles, carrying out controlled cord traction and carrying out uterine massage. Findings are presented in Table 2.3 and indicate that the vast majority of ANMs (75-93\%) had received training in conducting these assessments. A little over half of the ANMs reported that they had received training in conducting all of the assessments. Rural-urban differences were narrow.

\section{Table 2.3}

Special training received for providing maternal health services

\begin{tabular}{|l|c|}
\hline Elements of training & $\mathrm{N}=96$ \\
\hline Measuring blood pressure & 75.0 \\
\hline Checking pallor & 84.7 \\
\hline Measuring fundal height & 77.5 \\
\hline Determining foetal presentation & 86.1 \\
\hline Conducting a normal delivery & 93.1 \\
\hline Giving uterotonic drug to enhance contraction of uterine muscles & 79.2 \\
\hline Carrying out controlled cord traction & 83.3 \\
\hline Carrying out uterine massage & 81.9 \\
\hline All of the above & $\mathbf{5 3 . 1}$ \\
\hline
\end{tabular}




\section{CHAPTER 3}

\section{Awareness of maternal and newborn care practices among ANMs}

Several questions were posed to ANMs about their awareness of maternal and newborn care practices. Five themes, namely, awareness of antenatal care practices, birth preparedness, postpartum care practices, newborn care practices, and complications during pregnancy, delivery, the postpartum period, and among newborns, were included.

\section{Awareness of antenatal care practices}

ANMs were asked detailed questions about their awareness of the number of antenatal check-ups that a woman should have, the appropriate timing of the first, second and third check-ups, the number of doses of tetanus toxoid injection to be taken and the number of iron and folic acid tablets to be taken. Awareness of the number of antenatal check-ups that a pregnant woman should have was universal, with 99\% (all but one) of ANMs reporting that a woman should have 3-4 antenatal check-ups. So was awareness of the appropriate timing of the first antenatal check-up; 97\% of ANMs knew that a woman should have her first antenatal check-up in the first trimester. However, awareness of the appropriate timing of the second and third checkups was somewhat limited. Over four-fifths of the ANMs knew that a woman should have her second antenatal check-up in the second trimester and about three-fifths knew that a woman should have her third antenatal check-up in the 8th month. Awareness of the number of doses of tetanus toxoid injections that a pregnant woman should have was also universal; 99\% of ANMs knew that a pregnant woman should receive two doses of TT. While 98\% of ANMs knew that a pregnant woman should consume 90-100 IFA tablets, somewhat fewer knew that a pregnant woman who is anaemic should be given 200 IFA tablets. Findings also indicate that

Table 3.1

Awareness of antenatal care practices

\section{$\%$ of ANMs who knew that:}

A woman should have 3-4 antenatal check-ups

A woman should have her first antenatal check-up in the first trimester

A woman should have her second antenatal check-up in the second trimester

A woman should have her third antenatal check-up in the 8th month

A woman should have 2 doses of tetanus toxoid injections

A woman should consume 90-100 iron and folic acid tablets

A woman who is anaemic should be given at least 200 iron and folic acid tablets

Correctly aware of all of the above

\section{$N=96$}

99.0

96.9

86.5

59.4

99.0

97.9

86.5

51.0 
only half of the ANMs were informed about all of the above.

Rural-urban differences were narrow on most indicators. However, a larger proportion of ANMs in urban than rural areas knew that a pregnant woman should have her third antenatal check-up in the 8th month (17 out of 24 versus 40 out of 72 ) and that a pregnant woman who is anaemic should be given 200 IFA tablets (23 out of 24 versus 60 out of 72). They were also more likely than their rural counterparts to have answered correctly all of the seven questions related to antenatal care practices posed in the study (16 out of 24 versus 33 out of 72).

\section{Awareness of birth preparedness and selected protocols to be followed during labour and delivery}

The study explored ANMs' awareness of birth preparedness by asking them to name the essential preparations that a woman should make for a safe delivery. All those who reported that a woman should identify a hospital or trained midwife for delivery, make arrangements for transportation, save money for delivery expenses, identify a referral health facility to attend in case of emergency or keep items for safe delivery in case she opts for a home delivery were considered to have some awareness of birth preparedness. Also probed were ANMs' awareness of standard protocols to be followed during labour and delivery, including not conducting a vaginal examination if the woman is bleeding during labour $^{1}$, not using oxytocin to speed up delivery, and using oxytocin or misoprostol for the active management of the third stage of labour.

Findings indicate that the majority of ANMs were aware of some of these essential birth preparations; for example, four-fifths identified making arrangements for transportation, three-fifths identified saving money for delivery expenses and half mentioned identifying a hospital or trained midwife for delivery as essential for a safe delivery. A little over two-fifths of the ANMs were aware of three or more preparations for a safe delivery. Rural-urban differences indicate that a larger proportion of ANMs in rural areas than urban areas identified three or more preparations for a safe delivery (34 out of 72 versus 8 out of 24).

Awareness of standard protocols to be followed during labour and delivery was limited. For example,

\section{Table 3.2}

Awareness of birth preparedness and selected protocols to be followed during labour and delivery

\begin{tabular}{l|r|}
\hline$\%$ of ANMs who knew that a woman should make the following & N=96 \\
preparations for a safe delivery: & 51.0 \\
\hline Identify a hospital or trained midwife for delivery & 81.3 \\
Make arrangements for transportation & 61.5 \\
Save money for delivery expenses & 8.3 \\
Identify a referral health facility to attend in case of emergency & 12.5 \\
Keep items for safe delivery in case she opts for a home delivery & 43.8
\end{tabular}

\footnotetext{
${ }^{1}$ We note that conducting vaginal examination is not recommended if the woman is bleeding during pregnancy beyond 12 weeks of pregnancy or at the time of labour (Ministry of Health and Family Welfare, 2010). In our study, we only probed ANM's awareness of the protocol that vaginal examination should not be conducted if the woman is bleeding at the time of labour.
} 
just two-thirds of the ANMs knew that a vaginal examination should not be conducted if the woman is bleeding at the time of labour, four-fifths knew that it is not good to give an oxytocin injection before the delivery and half knew that the use of oxytocin or misoprostol for the active management of the third stage of labour is recommended. Indeed, just one-third of the ANMs were correctly informed about all three protocols, with somewhat more rural than urban ANMs reporting so (23 out of 72 versus 6 out of 24).

\section{Awareness of postpartum care practices}

The study also assessed the extent to which ANMs were aware that a woman should have her first postpartum checkup within a few hours or at best within two days of delivery and that a woman should have at least three postpartum check-ups. Findings show that the majority of ANMs were aware of postpartum care practices; four-fifths of the ANMs knew that a woman should have her first check-up within a few hours or at best within two days of delivery (80\%); rural-urban differences were narrow. However, fewer knew that a woman should have three postpartum check-ups (52\%); more rural than urban ANMs reported so (40 out of 72 versus 10 out of 24).

Figure 3.2: Awareness of postpartum care practices $\%$ of ANMs who knew that $(\mathrm{N}=96)$

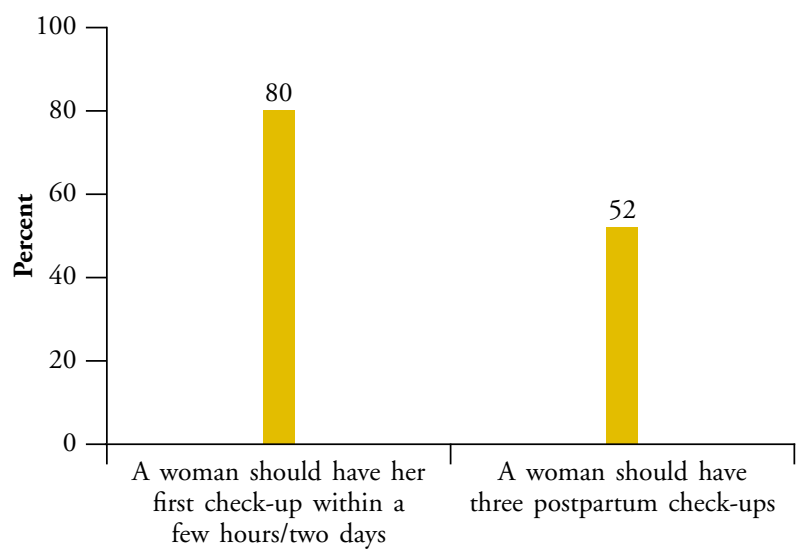

\section{Awareness of newborn care practices}

The study explored ANMs' awareness of several dimensions of newborn care practices, including the immediate care of the newborn, feeding practices and immunization. With regard to immediate care

Figure 3.1: Percentage of ANMs who were aware of the protocols to be followed during labour and delivery

$\%$ of ANMs who were aware of the selected protocols ( $\mathrm{N}=96)$

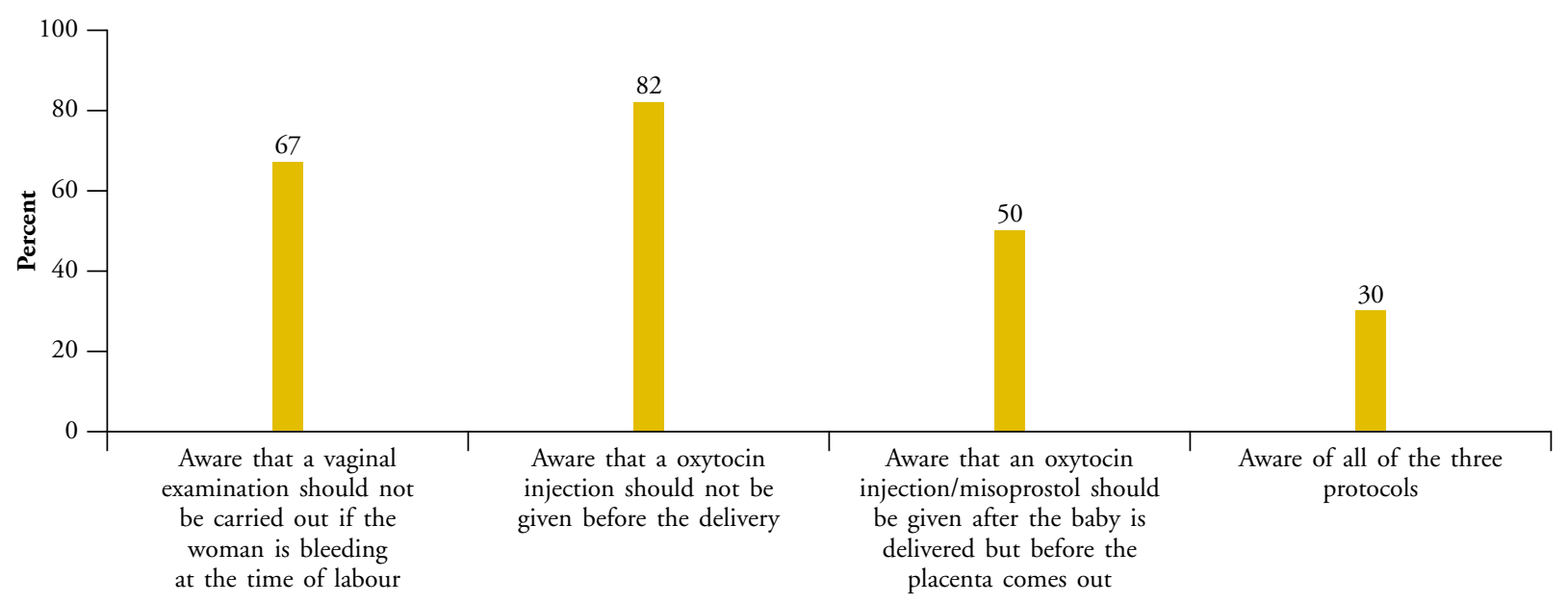


of the newborn, the study assessed whether ANMs knew that the umbilical cord should be cut after 2-3 minutes of delivery, a newborn should be wiped dry and not bathed immediately after birth and that it is not good to apply any substance on the cord stump. A summary indicator of comprehensive awareness of best practices regarding immediate care of the newborn, reflecting the percentage of ANMs who were knowledgeable about all three aspects, was also calculated. In the area of feeding practices, the study assessed whether ANMs were aware that a newborn should be breastfed within an hour of delivery, fed colostrum, exclusively breastfed for six months and offered complementary food at six months of age. An indicator of comprehensive awareness of feeding practices, as measured by percentages aware of all four aspects, was also calculated. To assess
ANMs' awareness of the immunization of infants, respondents were asked about the number of doses of BCG, oral polio, DPT and measles vaccines to be given to an infant as well as about the timing of different doses of BCG, oral polio and DPT vaccines to be given.

Findings show that the vast majority of ANMs were aware of best practices related to the immediate care of the newborn. For example, 93\% of ANMs knew that the umbilical cord should be cut after 2-3 minutes of delivery, 99\% knew that a newborn should be wiped dry and not bathed immediately and that $96 \%$ knew that it is not good to apply any substance on the cord stump. Some $88 \%$ of ANMs displayed awareness of all three practices; rural-urban differences were narrow.

\section{Table 3.3}

\section{Awareness of newborn care practices}

\section{$\%$ of ANMs who knew that:}

The umbilical cord should be cut within 2-3 minutes of delivery

A newborn should be wiped dry and not be bathed immediately

It is not good to apply any substance on the cord stump

Comprehensive awareness of best practices related to the immediate care of the newborn

A newborn should be breastfed within an hour of delivery

A newborn should be fed colostrums

An infant should be breastfed exclusively for 6 months

An infant should be offered complementary food at six months of age

Comprehensive awareness of feeding practices

A newborn should be given one dose of BCG vaccine

A newborn should be given BCG vaccination at birth

A newborn should be given 4 doses of oral polio vaccine till age 1

A newborn should be given the first dose of oral polio vaccine at birth

A newborn should be given second dose of oral polio at 6 weeks

A newborn should be given three doses of DPT vaccine

A newborn should be given the first dose of DPT vaccine at six weeks

A newborn should be given a single dose of measles vaccine

Comprehensive awareness of immunization

\section{$\mathrm{N}=96$}

92.7

99.0

94.8

87.5

100.0

97.9

96.9

93.8

91.7

100.0

100.0

78.1

97.9

97.9

93.8

100.0

100.0

75.0 
Awareness of feeding practices, likewise, was high; almost all the ANMs (94-100\%) knew that a newborn should be breastfed within an hour of delivery and should be fed colostrum, and that an infant should be breastfed exclusively for six months and offered complementary food at six months of age. Over $90 \%$ of ANMs displayed comprehensive awareness of feeding practices; rural-urban differences were again narrow.

Awareness of the immunization schedule, similarly, was high. Almost all the ANMs (94-100\%) were correctly informed about the number of doses of BCG, DPT and measles vaccines to be given to an infant as well as about the ages at which different doses of these vaccines are to be given. Somewhat fewer ANMs, just four-fifths, knew that a newborn should be given 4 doses of oral polio vaccine in infancy.

Comprehensive awareness of the immunization schedule, that is, correct awareness of the range, dosage and timing of vaccines to be given to an infant, was, however, limited. Just three-quarters of the ANMs were correctly informed about all of these issues. A larger proportion of ANMs in rural than urban areas displayed comprehensive awareness of immunization schedules for infants (57 out of 72 versus 15 out of 24$)$.

\section{Awareness of complications during pregnancy, delivery and the postpartum period, and among newborns}

We assessed ANMs' awareness of maternal and newborn complications based on their responses to four multiple response questions in which we asked them to list all the warning signs during pregnancy, delivery, and the postpartum period, and among newborns, respectively, for which a woman should seek care from a health care provider. ANMs who spontaneously listed the following symptoms—-severe headache, blurred vision, high blood pressure, swelling around ankles, puffiness of face, fits, high fever, vaginal bleeding, foul-smelling vaginal discharge, jaundice and anaemia - as warning signs during pregnancy were assumed to have some awareness of complications during pregnancy. Likewise, ANMs who listed such symptoms as abnormal foetal presentation, prolonged labour, obstructed labour, heavy bleeding, fits and retained placenta as warning signs during labour and delivery were assumed to have some awareness of complications during labour and delivery. Similarly, all those who listed such symptoms as heavy bleeding, high fever, foul-smelling vaginal discharge and fits as warning signs during the postpartum period were assumed to be aware of postpartum complications. Finally, ANMs who listed the following symptoms-difficulty in breathing, fast breathing, high fever, difficulty in suckling, stiffness of the body, no bowel movement or urination within 24 hours, infected umbilical cord, diarrhea and jaundice-were assumed to be aware of complications in newborns.

Findings presented in Table 3.4 show that the majority of ANMs were aware of complications during pregnancy; $75 \%$ were able to list at least three severe complications during pregnancy. Anaemia followed by swelling around ankles and puffiness of face, and severe headache, blurred vision or high blood pressure, were most frequently cited and foul-smelling vaginal discharge followed by jaundice were least frequently cited. Fewer were aware of complications during labour and delivery; $32 \%$ of ANMs cited three or more danger signs during labour and delivery. Even fewer were aware of complications during the postpartum period; $19 \%$ of ANMs cited three or more danger signs 
Table 3.4

Awareness of complications during pregnancy, delivery and the postpartum period, and among newborns

\section{$\%$ of ANMs who spontaneously mentioned the following as complications: $\quad$ N=96}

\section{During pregnancy}

Severe headache/blurred vision/high BP

67.7

Swelling around ankles/puffiness of face

68.8

Fits

High fever

Vaginal bleeding

Foul-smelling vaginal discharge

Jaundice

10.4

Anaemia

79.2

Reported 3 or more of the above

During labour and delivery

Abnormal foetal presentation

Labour too long

39.6

Obstructed labour

Fits

30.2

Heavy bleeding

76.0

Placenta not coming out

Reported 3 or more of the above

\section{During the postpartum period}

Heavy bleeding

High fever

Bad smelling vaginal discharge

14.6

Fits

Reported 3 or more of the above

Among newborns

Difficulty in breathing

Fast breathing

High fever

51.6

Difficulty in suckling

Stiffness of the body

No bowel movement or urination within 24 hours

Infected umbilical cord

Diarrhoea

21.1

Jaundice

27.4

Reported 3 or more of the above

54.2 
during the postpartum period. With regard to awareness of complications among the newborns, slightly over half of the ANMs cited three or more severe complications. A larger proportion of ANMs in rural than urban areas were aware of three or more severe complications that can occur during the antenatal period (55 out of 72 versus 17 out of 24); however, a reverse pattern was observed with regard to awareness of three or more severe complications during delivery ( 21 out of 72 versus 10 out of 24 ) and the postpartum period (12 out of 72 versus 6 out of 24). Rural-urban differences were narrow with respect to ANMs' awareness of complications in the newborns.

The study also asked about ANMs' awareness of an appropriate health facility for treating selected pregnancy related complications such as the experience of puffiness of the face, antepartum bleeding, i.e., bleeding occurring after 20 weeks of pregnancy or during labour but before the delivery of the baby and prolonged labour. Findings presented in Table 3.5 indicate that awareness of an appropriate health facility for treating pregnancyrelated complications was somewhat limited among ANMs. Findings indicate that sizeable proportions of
ANMs would refer women experiencing pregnancyrelated complications to a facility that was not equipped to address the complications. For example, 54\% ANMs reported that a pregnant woman who experiences puffiness of the face can be treated in a sub-centre; $35 \%$ and $42 \%$ of ANMs reported that a pregnant woman experiencing antepartum bleeding and prolonged labour can be treated in a primary health centre, respectively; the recommended lowest level facility for these are a primary health centre and a community health centre, respectively. A larger proportion of ANMs in rural than urban area were found to refer women reporting these complications to a facility not equipped to address the complications ( 32 out of 72 versus 2 out of 24 referring a woman experiencing antepartum bleeding to a primary health centre, for example). Only 33-46\% of ANMs reported that they would refer women experiencing these complications to the recommended facility. A larger proportion of ANMs in rural than urban areas were found to refer women to the recommended facility (39 out of 72 versus 5 out of 24 referring a woman experiencing antepartum bleeding to an FRU, for example). Some13-22\% of ANMs reported that they would

\section{Figure 3.3: Percentage of ANMs who spontaneously mentioned the complications during pregnancy,} delivery and postpartum period

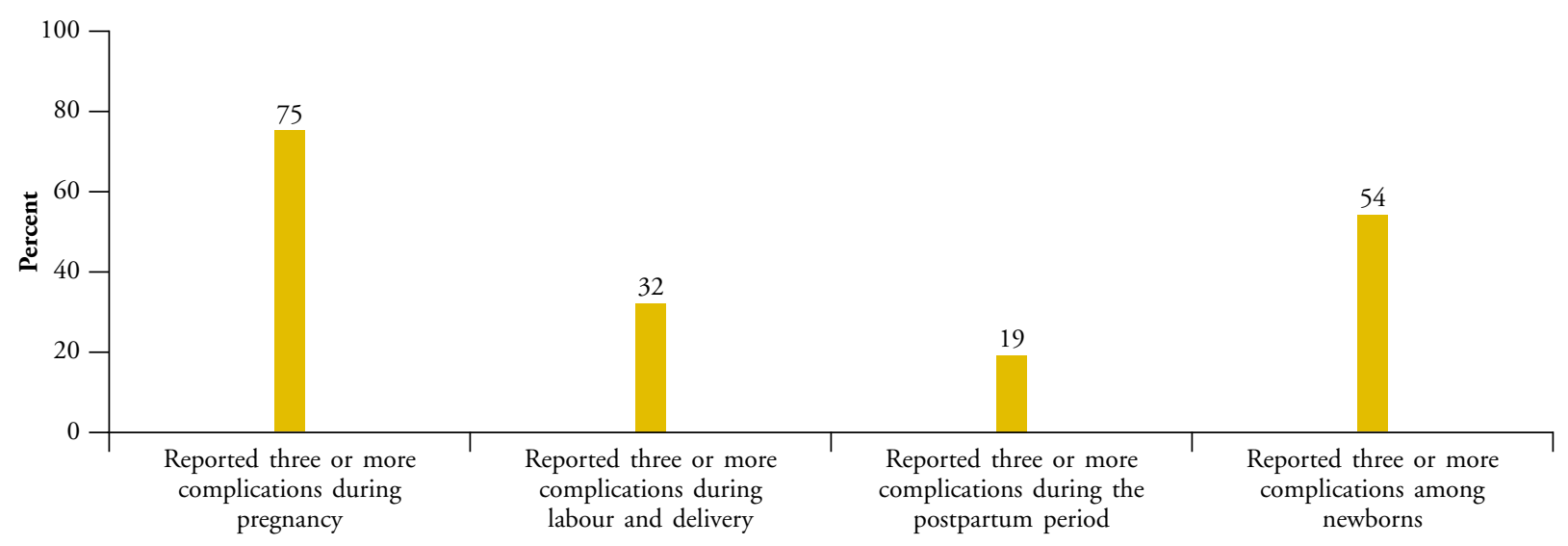




\section{Table 3.5}

ANMs' awareness of recommended facilities to which to refer a pregnant woman who experiences selected pregnancy-related complications

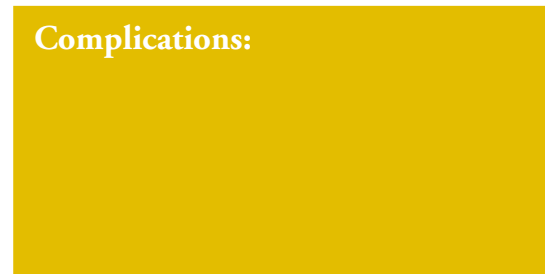

\begin{tabular}{|c|c|c|}
\hline $\begin{array}{c}\text { \% of ANMs who would refer pregnant women experiencing selected } \\
\text { complications to }(\mathrm{N}=96)\end{array}$ \\
\hline $\begin{array}{c}\text { A facility not equipped } \\
\text { to address this } \\
\text { problem }\end{array}$ & $\begin{array}{c}\text { Recommended facility } \\
\text { (lowest level of facility } \\
\text { equipped to address } \\
\text { the problem) }\end{array}$ & $\begin{array}{c}\text { Higher level facility } \\
\text { (equipped to address } \\
\text { the problem, but not } \\
\text { necessarily required) }\end{array}$ \\
\hline
\end{tabular}

Puffiness of the face

(Recommended: PHC)

54.2

33.3

12.5

Antepartum bleeding

(Recommended: FRU/CHC)

35.4

45.8

18.8

Labour lasting more than 12 hours

(Recommended: FRU/CHC)

41.6

36.5

21.9

refer such cases to a higher level facility, although a referral to such facilities was not required; for example, referring women experiencing puffiness of the face to a community health centre or district hospital and those experiencing antepartum bleeding and prolonged labour to a district hospital. A larger proportion of urban than rural ANMs so reported, perhaps, because of easier access to higher level facilities in urban than rural areas (17 out of 24 versus 1 out of 72 referring a woman experiencing antepartum bleeding to a district hospital, for example).

Finally, the study also assessed ANMs' awareness of proper management of selected obstetric complications, such as pre-eclampsia in a woman whose delivery is imminent and retained placenta in a woman who has delivered at home. Findings, presented in Table 3.6, suggest limited awareness, among ANMs, of proper management of obstetric complications. For example, only $16 \%$ of ANMs reported that if a pregnant woman who experiences pre-eclampsia and whose delivery is imminent approaches the ANM, she should conduct the delivery rather than refer the woman to an FRU without conducting the delivery. Rural ANMs were more likely than urban ANMs to report so (14 out of 72 versus 1 out of 24 ). Likewise, only $70 \%$ of ANMs reported that if a woman whose delivery was attended

\section{Table 3.6}

Awareness of proper management of selected pregnancy-related complications

\section{$\%$ of ANMs who knew that:

If a pregnant woman who experiences pre-eclampsia and whose delivery is imminent approaches the ANM, she should conduct the delivery rather than refer her to an FRU without conducting the delivery

If a woman whose delivery was attended by the ANM at home has not expelled the placenta within an hour of delivery of the baby, the ANM must refer the woman to a medical officer at the PHC rather than remove the placenta manually at home 
by the ANM at home had not expelled the placenta within an hour of delivery of the baby, she must refer the woman to a medical officer at the PHC rather than remove the placenta manually at home; urban ANMs were more likely than rural ANMs to so report (21 out of 24 versus 46 out of 72 ). 


\section{CHAPTER 4}

\section{Services provided by ANMs}

The study also probed the maternal and newborn care related services that ANMs had provided to women in their community in the three months preceding the interview, and the extent to which they had followed the standard protocols of care while delivering these services. Findings are described in this chapter.

\section{Antenatal care-related services}

Almost all the ANMs (98\%) reported that they had provided antenatal care-related services to pregnant women in the three months preceding the interview. On average, an ANM provided such services to 25 women in the three months preceding the interview (not shown in table).

Several questions were posed to assess the quality of antenatal care-related services provided by ANMs. Specifically, we probed about the extent to which ANMs had followed standard protocols while conducting antenatal check-ups-taking a medical history whenever a pregnant woman comes for her first antenatal check-up, conducting a physical examination as part of an antenatal check-up, conducting basic laboratory investigations, reminding women about the timing of their second dose of TT injection, discussing the side effects of taking medicines, if prescribed, and enquiring about signs of complications experienced. We also asked them about the extent to which they had maintained auditory and visual privacy for the clients while providing antenatal care-related services and about the nature of interactions with their clients. We note that the chances of respondent bias cannot be ruled out.
Table 4.1 presents findings related to ANMs' perspectives on the extent to which they had followed the standard protocols while conducting antenatal check-ups. Almost all the ANMs (97\%) reported that they always took a medical history whenever a pregnant woman came for her first antenatal check-up. Information collected while taking a medical history included the woman's experience of any previous pregnancies (83\%), the date of her last menstrual period (52\%), any history of systemic illness (50\%) and symptoms experienced during pregnancy (43\%). Over 90\% of ANMs reported that they always conducted a physical examination whenever a pregnant woman came for an antenatal check-up. The most commonly conducted examination was checking a pregnant woman's weight, reported by $81 \%$ of ANMs. Checking of blood pressure and conducting an abdominal examination was reported by $62 \%$ and $56 \%$ of ANMs, respectively. Fewer ANMs reported that they checked pallor (41\%) and measured the height of the woman (38\%). No more than half of the ANMs reported that they conducted laboratory investigations as part of antenatal services provided; these investigations largely comprised of checking haemoglobin, reported by $44 \%$ of ANMs. Investigations to assess albumin or sugar in urine were reported by $19 \%$ and $8 \%$ of ANMs, respectively. All the ANMs reported that they always reminded the pregnant woman to come back for the second dose of TT injection, 94\% reported that they always discussed the side effects of taking medicines, if prescribed, and $98 \%$ reported that they always enquired about signs of complications experienced 
by pregnant woman. However, only $42 \%$ of ANMs reported that they had followed all of the above protocols while conducting antenatal check-ups. Rural—urban differences were, by and large, narrow. However, a larger proportion of rural than urban ANMs conducted laboratory investigations (36 out of 72 versus 10 out of 24).

Although not exactly comparable, we note some inconsistency between the reports of the ANMs and of the women who had delivered in the one year preceding the interview in the same study villages and urban wards in which the ANMs were practising. Findings from a companion study of women who had received antenatal services in public sector facilities (village clinics organized by ANMs, sub-centres, primary health centres, urban health centres, community health centres and other government facilities) show that only $62 \%, 42 \%$ and $18 \%$ of women reported that the provider had always reminded them about follow-up visits, asked about signs of complications experienced by them and discussed the side effects of taking prescribed medicines, respectively (Santhya et al., 2011). In comparison, $94-100 \%$ of ANMs reported that they routinely followed all three practices. This inconsistency in reporting may be because the ANMs may have overestimated their contribution.

\section{Table 4.1}

Percentages of ANMs reporting that they had followed standard protocols while conducting antenatal check-ups in the three months preceding the interview

\section{Indicators of standard protocols}

Always took a medical history whenever a pregnant woman came for her first antenatal check-up

Inquired about any previous pregnancies

Inquired about the date of the last menstrual period

Inquired about any history of systemic illnesses

Inquired about symptoms experienced during pregnancy

Always conducted physical examinations as part of ANC services

Measured weight

Checked blood pressure

Conducted abdominal examination

Checked pallor

Measured height

Conducted laboratory investigations as part of ANC services

Checked haemoglobin

Checked albumin in urine

Checked sugar in urine

Always reminded the pregnant woman to come back for the second dose of TT injection

Always discussed the side effects of taking prescribed medicines, if prescribed

Always asked about signs of complications experienced by the pregnant woman

Followed all of the above
$\mathrm{N}=96$

96.9

83.3

52.0

50.0

42.7

92.7

81.3

61.5

56.3

40.6

37.5

47.9

43.8

18.8

8.3

100.0

93.8

97.9

41.7 
Figure 4.1 summarises findings related to the extent to which the ANMs had maintained auditory and visual privacy for women while providing antenatal care-related services and the nature of their interactions with the women. Findings indicate that auditory privacy is rarely maintained; just $9 \%$ of ANMs reported that they ensured always or most of the time that no one could overhear their interactions with the pregnant woman in the facility. Moreover, visual privacy was not maintained by all the ANMs; just $78 \%$ of ANMs reported that they ensured always or most of the time that no one could see when she examined a pregnant woman. A larger proportion of ANMs in rural than urban areas reported that they had maintained auditory privacy (9 out of 72 versus none) and visual privacy (59 out of 72 versus 16 out of 24) for clients. Almost all the ANMs (98\%) reported that they usually asked the woman whether she had any questions to discuss with them and 92\% reported that that they usually spent at least 10-15 minutes in consultation with the pregnant woman. Rural-urban differences were narrow.
We note from the companion study of women in the study area that women's reports of the extent to which the health care providers had maintained privacy during consultations, and the nature of their interactions, differed from that of the ANMs'. For example, of women who had received antenatal services in public sector facilities, 35\% and 73\% reported that the provider had always or most often maintained auditory and visual privacy, respectively, while conducting antenatal check-ups (compared to $9 \%$ and $78 \%$ of ANMs). Likewise, $40 \%$ and $54 \%$ of women reported that the provider typically asked them whether they had any questions to discuss with them and spent 10-15 minutes with them in consultation (Santhya et al., 2011), compared to $92 \%$ and $30 \%$ of ANMs so reporting, respectively.

About one-third of the ANMs reported that pregnant women took their advice seriously (30\%), while the rest felt that the women took their advice somewhat seriously. Somewhat more rural than urban ANMs reported that women took their advice seriously (23 out of 72 versus 6 out of 24).

Figure 4.1: Percentages of ANMs reporting that they maintained privacy for women and had good interactions with women

Indicators of quality of antenatal care-related services $(\mathrm{N}=96)$

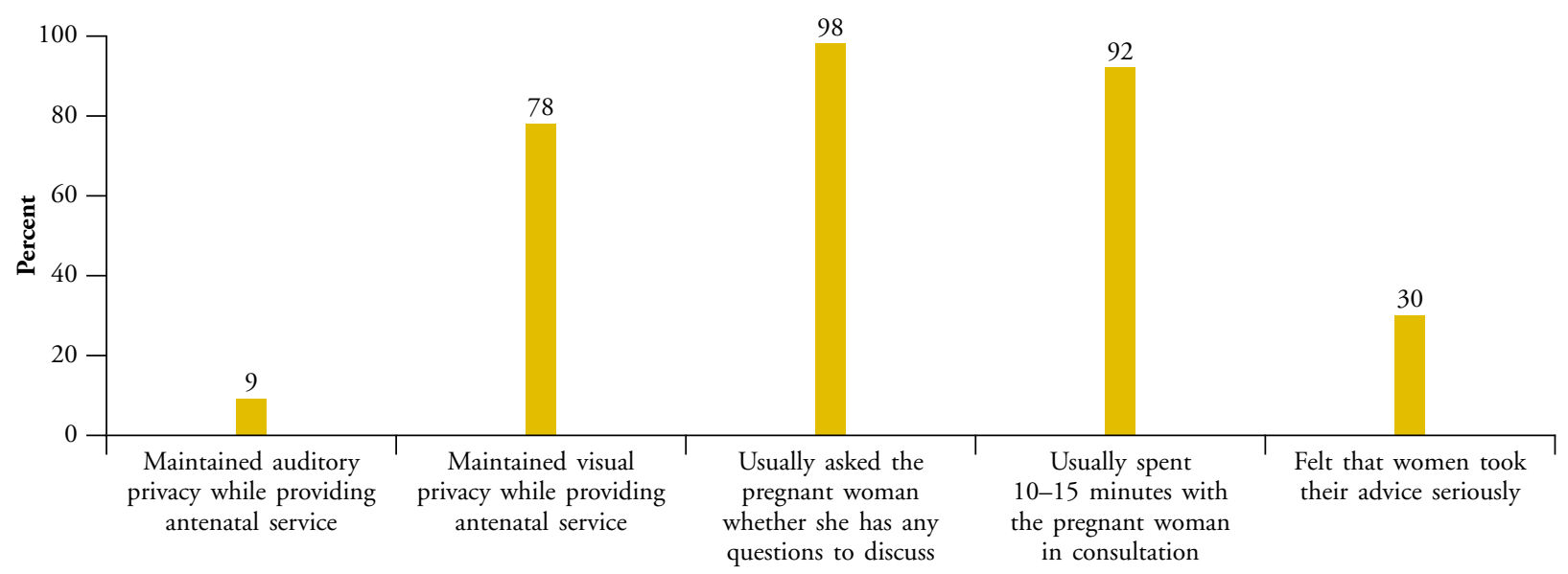




\section{Delivery care-related services}

Several questions were posed to the ANMs to assess the extent to which they had rendered delivery care-related services. Findings are presented in Table 4.3. Almost all the ANMs (98\%) reported that they had assisted pregnant women in identifying a health facility for delivery in the three months preceding the interview. Rural-urban differences were negligible. ANMs who reported so had, on average, assisted 20 pregnant women in the three months preceding the interview (not shown in table).

A little over one-third of the ANMs had conducted deliveries in the last three months (Figure 4.2). ANMs who reported so had, on average, conducted four deliveries in the last three months. A larger proportion of ANMs in rural than urban areas had conducted deliveries in the last three months (32 out of 72 versus 3 out of 24). Of 35 ANMs who had conducted deliveries, 13 had conducted deliveries at home at least once; 18 at the sub-centre at least once and 9 at a primary health centre or community health centre at least once in the three months preceding the interview (not shown in Figure).

Figure 4.2: Delivery care-related services provided by ANMs

Type of services $(\mathrm{N}=96)$

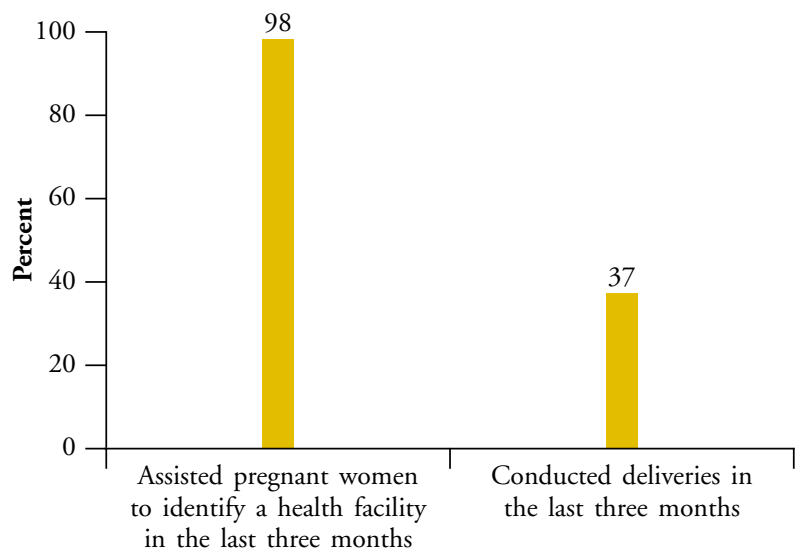

The study asked ANMs, who reported that they had conducted deliveries in the three months preceding the interview, about the quality of delivery services. Specifically, we probed the extent to which they had followed standard protocols related to delivery care: (1) conducting essential examinations, namely, abdominal and vaginal examinations, and temperature and pulse measurement, when a woman was admitted to the facility for delivery; (2) conducting vaginal examinations about once in four hours during labour and delivery, and not more frequently; (3) using clean gloves while conducting vaginal examinations; (4) washing hands with soap and water before and after conducting vaginal examinations; (5) using disposable syringes; (6) using the partograph; and (7) giving oxytocin for the active management of the third stage of labour. We also asked them whether they abstained from harmful practices during labour and delivery, namely, (1) exerting strong fundal pressure before the woman delivered; and (2) injecting oxytocin to speed up the delivery or increase pain. Finally, we included some questions related to the quality of services at the facility and the treatment of women at the facility. We define good quality services and treatment as those that: (1) do not ask the woman or her family members to arrange medicine and other supplies; (2) ensure visual privacy for the woman during delivery; (3) discharge the woman at least 24 hours after delivery; and (4) allow the escort to remain with the woman during delivery. We caution that only 35 of the 96 ANMs who participated in the study had conducted the deliveries and given these small numbers, findings need to be interpreted with caution. Moreover, response bias cannot be ruled out. Findings are presented in Table 4.2.

With regard to the deliveries they had conducted in the three months prior to the interview, almost all the ANMs reported that they had conducted 
all the essential examinations whenever a woman was admitted to their facility for delivery (33 out of 35), always used clean gloves while conducting vaginal examinations, washed hands before and after conducting vaginal examinations, and used disposable syringes. However, just one ANM reported that she had conducted vaginal examinations during labour and delivery about once in four hours and not more frequently. Likewise, just two ANMs reported that they always used the partograph and eight reported that they always or sometimes gave oxytocin to manage the third stage of labour. With regard to abstaining from harmful practices during labour and delivery, findings present a mixed picture. For example, 31 out of 35 ANMs reported that they never applied strong fundal pressure before the woman delivered; however, 18 of the 35 ANMs reported that they gave intra-muscular injection (most likely oxytocin) during labour before the baby was delivered to speed up the delivery/increase pain. Findings were mixed with respect to the treatment of the woman at the facility as well. All the ANMs reported that they ensured visual privacy for the woman during delivery. The majority of the ANMs reported that they discharged the woman at least after 24 hours of delivery (23 out of 35). However, more than half of the ANMs reported that they had asked the woman or her family to arrange medicine and other supplies (16 out of 35), and less than half of the ANMs or allowed the escort to remain with the woman during delivery (14 out of 35). Most ANMs who discharged the woman soon after delivery argued that they had done so because the woman or her family members had insisted on her early discharge, or because the health centre lacked facilities to keep the delivered woman longer at the facility.

As observed in the case of antenatal care practices, we note that ANMs' reports of the extent to which they had followed the standard protocols of care differed on several counts from reports of women highlighted in a companion study. For example with regard to following healthy practices during labour and delivery, women's reports were not as positive as those of ANMs. Only 24\% of women who delivered in government facilities reported that the provider had conducted all the essential examinations when they were admitted to the facility for delivery; 59\% reported that the provider had always washed her hands before and after conducting vaginal examination. In sharp contrast, almost all the ANMs reported that they routinely followed both of these practices (33 and 34 of 35 ANMs, respectively). Likewise, while $9 \%$ of women who delivered in government facilities reported that they had been given an injection after the baby was delivered but before the placenta was expelled (most likely oxytocin, for the management of the third stage of labour), somewhat more ANMs (8 of 35) reported this practice. The only indicator for which the women's and the ANMs' reports matched was the use of clean gloves for conducting vaginal examinations; almost all the women and the ANMs reported that they used clean gloves. As regards harmful practices during labour and delivery, both the ANMs and the women acknowledged the pervasiveness of these practices; half of the ANMs and three-quarters of the women reported the use of intra-muscular injection (most likely, oxytocin) to speed up the delivery or increase labour pain, for example. Inconsistency in ANMs' and women's reports was apparent with regard to indicators of women's treatment at the facility as well. While more than half of the ANMs reported that they had asked the women or their family members to arrange for medicines and other supplies, $86 \%$ of women so reported. Likewise, while all the ANMs reported that they ensured visual privacy for women during delivery, only $73 \%$ of women so reported (Santhya et al., 2011). 
Table 4.2

Percentage of ANMs reporting that they had followed standard protocols of delivery care and abstained. from harmful practices

\section{Indicators of standard protocols of delivery care}

Conducted all the essential examinations whenever a woman was admitted to the facility for delivery

Conducted vaginal examinations during labour and delivery at appropriate intervals (no more than about once in four hours)

Always used clean gloves while conducting vaginal examinations

Always washed hands before and after conducting vaginal examinations

Always used disposable syringes

Always used the partograph

Gave oxytocin injection always or sometimes after delivery but before the placenta came out

\section{Indicators of harmful practices}

Never applied strong pressure on the woman's abdomen before she delivered

Never gave an intra-muscular injection during labour before the baby was delivered to speed up the delivery/increase pain

\section{Indicators related to treatment at the facility}

Never asked the woman or her family to get medicines and other supplies from outside 16

Ensured visual privacy for the woman during delivery

Discharged the woman after 24 hours

Always allowed the accompanying person to remain with the woman during delivery

14

ANMs who had conducted deliveries in the three months preceding the interview were also asked whether they had come across any complicated cases which they were not able to manage. Of the 35 ANMs who had conducted deliveries, 13 had come across at least one complicated case in the three months. Complicated cases faced by the ANMs included prolonged labour, heavy bleeding, severe anaemia, twin births, and, most often, obstructed labour. Almost all the ANMs reported that they referred the complicated case to FRUs.

\section{Postpartum and newborn care-related services}

Almost all the ANMs reported that they had visited recently delivered women in the last three months
(Table 4.3); rural-urban differences were modest. ANMs, on average, had made postpartum visits to some 15 women in the last three months (not shown in table). Fewer had visited recently delivered women within two days of delivery (37\%); ANMs in urban areas were somewhat more likely than their rural counterparts to have done so (10 out of 24 versus 25 out of 72). ANMs, regardless of whether they had made a postpartum visit in the last three months, reported that they typically enquired about or checked vaginal bleeding (91\%), blood pressure $(34 \%)$, temperature $(24 \%)$ and uterine contraction (17\%) whenever they made a first postpartum visit to a recently delivered woman. For women who delivered at sub-centre, ANMs reported that they checked vaginal bleeding (56\%), 
blood pressure $(36 \%)$, uterine contraction $(23 \%)$ and temperature $(18 \%)$ before they discharged the women.

Almost all the ANMs reported that they usually counseled recently delivered women about postpartum care; rural-urban differences were narrow. The topics that most ANMs discussed with women included self-care $(92 \%)$, care of the newborn (85\%), and breastfeeding (80\%) (not shown in table). Some $85 \%$ of ANMs reported that they always counseled recently delivered women about using contraception; more urban than rural ANMs so reported (22 out of 24 versus 60 out of 72). Contraceptive methods recommended by
ANMs were most frequently non-terminal methods such as condoms (98\%), the IUD (82\%), and oral pills (78\%); fewer ANMs recommended female sterilization (48\%), male sterilization (32\%), safe period (5\%) and lactational amenorrhoea method (2\%) (not shown in table).

We also probed the extent to which ANMs had followed standard protocols related to newborn care practices: (1) not delivering the newborn on to the floor; (2) cutting the cord after 2-3 minutes of delivery; (3) not applying any substance on the cord stump; (4) always wiping the newborn dry and wrapping immediately instead of bathing the newborn immediately; (5) encouraging the mother

\section{Table 4.3}

Percentages of ANMs reporting that they had provided postpartum care-related services in the three months preceding the interview

\begin{tabular}{|l|c|}
\hline Type of services & N=96 \\
\hline Made postpartum visits to recently delivered women & 96.9 \\
\hline Usually made two or more visits to recently delivered women & 96.9 \\
\hline Usually made the first postpartum visit within 2 days of delivery & 36.5 \\
\hline Usually made the second postpartum visit within 7-10 days of delivery & 41.7 \\
\hline Usually counselled recently delivered women about care following delivery & 99.0 \\
\hline Always counselled recently delivered women about using contraception & 85.4 \\
\hline
\end{tabular}

\section{Table 4.4}

Percentage of ANMs reporting that they had followed standard protocols of newborn care in deliveries conducted in the three months preceding the interview

\section{Indicators of standard protocols of newborn care $\quad \quad N=35$}

Never delivered the newborn on to the floor 31

Usually cut the cord after $2-3$ minutes of delivery $\quad 32$

Never applied any substance on the cord stump 32

Always wiped the newborn dry and wrapped immediately and never allowed the newborn to be bathed immediately $\quad 35$

Always encouraged the mother to breastfeed the newborn immediately 35

Always encouraged the mother to feed colostrum to the newborn 34 
to breastfeed the newborn immediately; and (6) encouraging the mother to feed colostrum to the newborn. Findings are presented in Table 4.4. Almost all the ANMs reported that they followed the standard protocols related to newborn care practices. For example, out of the 35 ANMs who had conducted deliveries in the three months preceding the interview, 31 reported that they had never delivered the newborn on to the floor, 32 cut the cord after 2-3 minutes of delivery, 32 had never applied any substance on the cord stump, and 35 always wiped the newborn dry and wrapped him/her immediately, and had never allowed the newborn to be bathed immediately. Likewise, all the ANMs reported that they encouraged the mother to feed colostrum to the newborn and breastfeed the newborn immediately.

Some $93 \%$ of ANMs reported that they had provided immunization services to newborns in the last three months; rural-urban differences were modest (not shown in table). 


\section{CHAPTER 5}

\section{ANIM's awareness of and perspectives on the JSY}

The study probed, in detail, ANMs' awareness of the JSY. Specifically, it assessed the extent to which ANMs were aware of the criteria under which women can avail of the JSY benefits and the benefits to which women and ASHAs are entitled. It also assessed the perspectives of ANMs about changes in their job responsibilities, work load and utilization of maternal health services following the introduction of the JSY as well as any shortcomings in the implementation of the JSY and their suggestions for improving its implementation.

\section{Awareness of the eligibility conditions for availling of the JSY}

Findings presented in Table 5.1 indicate that almost all the ANMs were aware of most eligibility conditions for availing of JSY benefits. For example, almost all the ANMs (99-100\%) knew that a woman delivering in a government health facility or an accredited private facility is eligible for JSY benefits, with little rural-urban differences. However, just $28 \%$ of ANMs were aware of an accredited private health facility, although there are some 11 accredited private facilities in the study districts; more urban than rural ANMs were aware of an accredited private health facility (12 out of 24 versus 15 out of 72) (not shown in Table). Just $13 \%$ of ANMs-all from rural areas— knew that a woman delivering at home is eligible for JSY benefits only if she is aged 19 and above, belongs to a BPL household and is having her first or second delivery. This is not surprising given the limited communication efforts that have been made to inform the community at large about women's entitlement to receive cash benefits for home deliveries under certain conditions.

The fact that women could avail of JSY benefits, regardless of their age, parity and household economic status was widely known. For example, all the ANMs knew that a woman delivering in a health facility is eligible for JSY benefits even if she is rich, $93 \%$ knew that she can avail of JSY benefits for any number of births and $84 \%$ knew that she can avail of JSY benefits regardless of her age. Ruralurban differences were by and large narrow.

Awareness of the type of government health facility where a woman could deliver to avail of JSY benefits was also widespread. For example, all the ANMs knew that deliveries in primary health centres, community health centres and district hospitals are eligible for JSY benefits. However, only $41 \%$ of ANMs knew that deliveries in a normal sub-centre, i.e., a sub-centre that is not accredited to conduct deliveries, are ineligible for JSY benefits; more rural than urban ANMs so reported (34 out of 72 versus 5 out of 24$)$.

\section{Awareness of women's and ASHAs' entitlements under the JSY}

ANMs' awareness of monetary and non-monetary support to which women are entitled under JSY is 


\section{Table 5.1}

Awareness of eligibility conditions for availing of the JSY

\section{$\%$ of ANMs who knew that:}

A woman delivering in a government health facility is eligible

A woman delivering in an accredited private facility is eligible

A woman delivering at home is eligible, only if she meets selected conditions ${ }^{a}$

A woman delivering in a health facility is eligible even if she is rich

A woman delivering in a health facility may avail of JSY benefits for any number of births

A woman delivering in a health facility may avail of JSY benefits regardless of her age

Deliveries in normal, i.e., non-accredited sub-centres ${ }^{\mathrm{b}}$ are ineligible

Deliveries in primary health centres are eligible

Deliveries in community health centres are eligible

Deliveries in district hospitals are eligible

aIf she is aged 19 and above, belongs to a BPL household and is having her first or second delivery; ${ }^{b}$ As per the JSY guidelines, deliveries conducted in sub-centres, which are accredited by the stateldistrict authorities are considered to be institutional deliveries and therefore, women delivering in these centres are eligible for all cash assistance under the JSY. Accredited sub-centres are those that are located in government buildings, with such facilities as electricity, water, and other medical requirements for basic obstetric services including drugs, equipment, and services of trained midwives for the purpose of conducting normal deliveries.

somewhat limited (Table 5.2). For example, only $81 \%$ of ANMs knew that a woman in a rural area is entitled to Rs. 1,400 and that a woman in an urban area is entitled to Rs. 1,000 if she delivers in a government or accredited private facility. Even fewer (64\%) knew that women delivering at home who meet selected conditions are entitled to Rs. 500. Moreover, only 54\%, $64 \%$ and $42 \%$ of ANMs knew that women are entitled to receive support from ASHAs during pregnancy, delivery and the postpartum periods, respectively. Just $7 \%$ reported that a woman is entitled to obtain transportation to reach the facility.

Findings indicate considerable confusion among the ANMs' with regard to ASHA's entitlements for supporting women in availing of pregnancy related services associated with the JSY (Table 5.3). Only $17 \%$ and $30 \%$ of ANMs reported that ASHAs who assist pregnant women in accessing maternal

\section{Table 5.2}

Awareness of women's monetary and non-monetary entitlements under the JSY

\section{$\%$ of ANMs who knew that a woman is entitled under the JSY to:}

\section{$\mathrm{N}=96$}

Rs. 1,400 in rural areas and Rs. 1,000 in urban areas, if a woman delivers in a hospital

Rs. 500, if a woman delivers at home

Support from ASHA during pregnancy 


\section{Table 5.3}

Awareness of ASHA's entitlements under the JSY

\section{$\%$ of ANMs who knew that:}

The ASHA is entitled to Rs. 600 for assisting pregnant women in accessing maternal health services in rural areas

The ASHA is entitled to Rs. 200 for assisting pregnant women in accessing maternal health services in urban areas

health services, particularly institutional delivery, are provided an incentive amount of Rs. 600 and Rs. 200 in rural and urban areas, respectively. The remaining reported amounts ranging from Rs. 100 to Rs. 500, perhaps because the amount stipulated in the scheme is apportioned for different activities, for example, for arranging for the woman's transportation to the health facility at the time of delivery.

\section{ANMs' awareness of schemes other than the JSY}

The study also included questions to assess ANMs' awareness of schemes other than the JSY intended to promote safe motherhood, such as the 108 ambulance service and the special nutritional supplementation scheme (for example, the provision of 5 kilograms of ghee to recently delivered women). Almost all the ANMs (99\%) were aware of the ambulance service. However, fewer (69\%) were aware that the ambulance service is free of cost. Finally, almost all the ANMs (98\%) had heard about the special nutritional supplementation scheme. Rural-urban differences were narrow, except that more urban than rural ANMs reported that the ambulance service is free of cost (19 out of 24 versus 47 out of 72).

\section{ANMs' perceptions about the JSY}

In order to assess ANMs' perceptions about the JSY, we probed whether there have been any changes in ANMs' job responsibilities with the introduction of the JSY, any changes in the utilisation of maternal health services, any difficulties that they experienced

Figure 5.1: Awareness of schemes other than the JSY

$\%$ of ANMs who were aware of $(\mathrm{N}=96)$

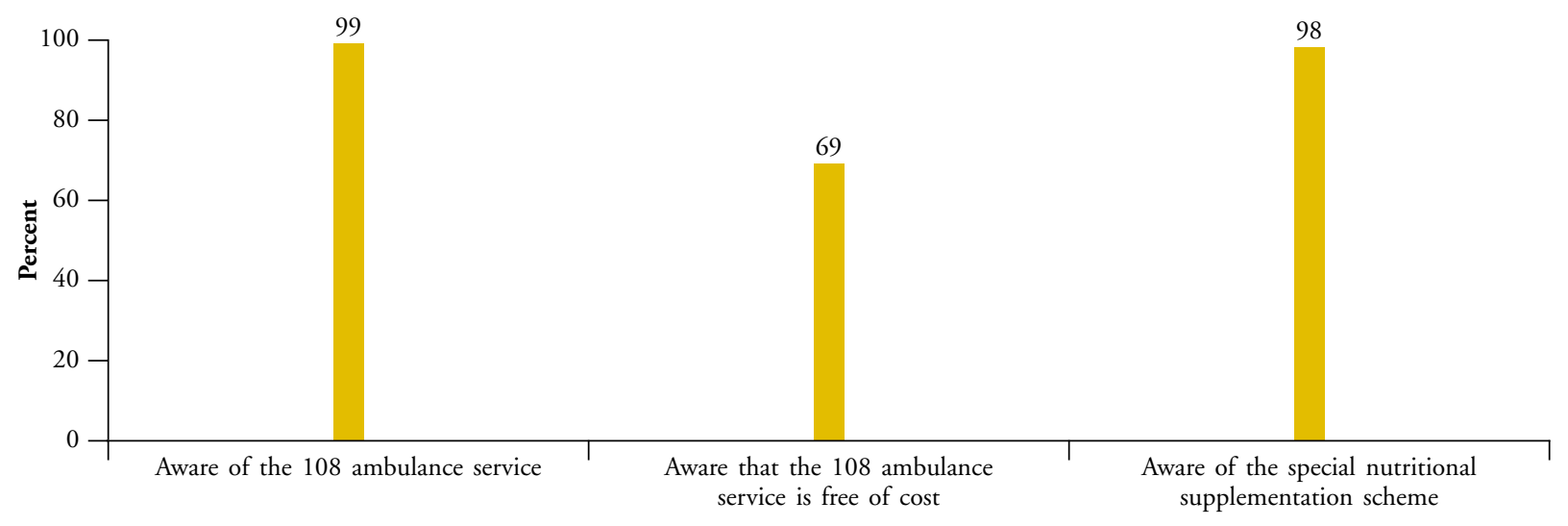


with increased utilisation of maternal health services, any shortcomings in the implementation of the JSY, and suggestions for improving the implementation of the JSY.

Over four-fifths of the ANMs believed that the job responsibilities of ANMs have changed with the introduction of the JSY. A larger proportion of rural ANMs than urban ANMs so reported (63 out of 72 versus 16 out of 24). Of those who reported that the job responsibilities of ANMs have changed, a large percentage mentioned that with the introduction of the JSY, ANMs were required to facilitate disbursement of cash to JSY beneficiaries $(72 \%)$ and submit reports relating to JSY implementation (64\%). A few mentioned such tasks as conducting training of ASHAs (10\%) and supervising ASHA's performance (8\%).

All or almost all the ANMs, regardless of rural-urban residence, believed that the utilisation of antenatal services, institutional deliveries and postpartum services had increased with the introduction of the JSY. Only 3\% of ANMs, all from rural areas, reported having experienced such difficulties as insufficient supplies and inadequate time to attend cases with the increased number of institutional deliveries. Almost all the ANMs reported that the workload of ANMs had increased; rural-urban differences were narrow.

About one-third of the ANMs said that there were shortcomings in the implementation of the JSY (not shown in Table). A range of concerns were raised, including those related to JSY cash payment and the quality of care in the health facility. Some ANMs observed that cash payments to women are delayed and that cheque payments are cumbersome. A few others were concerned about giving cash benefits to all women, irrespective of the number of children that women have and argued that the benefits should be restricted to a maximum of two children. Likewise, a few were concerned that the payment is linked only to institutional delivery rather than to the immunization of the infant as well; they argued that half of the amount should be paid if the woman undergoes an institutional delivery and the remaining half once the infant has been immunized. Issues related to incentive payments to ASHAs were also mentioned, including, for example, delayed payments to ASHAs and denying ASHAs part of their incentive by paying the transportation allowance to women directly if they made their own transportation arrangements to reach the facility. ANMs who mentioned shortcomings related to quality of care in the health facility listed such issues

\section{Table 5.4}

Percentage of ANMs reporting changes in job responsibilities and utilization of maternal health services with the introduction of the JSY

\begin{tabular}{|r|r}
\hline Indicator & $\mathrm{N}=96$ \\
\hline Job responsibilities of ANMs changed & 82.3 \\
\hline Utilisation of antenatal care services increased & 99.0 \\
\hline Institutional deliveries increased & 100.0 \\
Some difficulties were experienced with increasing number of institutional deliveries & 3.1 \\
Utilisation of postpartum services increased & 100.0 \\
Workload of ANMs increased & 95.8
\end{tabular}


as ill treatment of women, including harassment of beneficiaries by staff for a share of the JSY money (not clear whether they were referring to other ANMs or other cadre of providers), inadequate staff at the PHC including female physicians, and lack of facilities for conducting deliveries at the sub-centre.

ANMs who participated in the study made a number of suggestions for improving the implementation of the JSY scheme. Some ANMs suggested phased-in payments of JSY benefits, that is, some part before delivery, some at delivery and some after the immunization of infants; others suggested increasing the cash amount to women, particularly those from BPL households and restricting the benefits to women delivering up to a maximum of three children. Several ANMs called for improving the infrastructure at the sub-centre and primary health centre levels, upgrading all sub-centres to model sub-centres, recruiting more providers, including female physicians, and increasing access to transportation facilities. Finally, some suggested that incentive payments to ASHAs should be increased and that benefits to women be paid only if escorted by ASHAs. 


\section{CHAPTER 6}

\section{Summary and recommendations}

The study has brought forth several positive findings as well as areas that require attention with regard to improving the competence and performance of ANMs in Rajasthan.

\section{Summary}

\section{The majority of ANMs were trained in delivering maternal and newborn health services}

Almost all the ANMs had received training in imparting information pertaining to contraception, care during pregnancy, delivery and the postpartum period, and newborn care. Moreover, the vast majority of ANMs (75-93\%) had received training in conducting such tasks as measuring blood pressure, checking pallor, measuring fundal height, determining foetal presentation, conducting a normal delivery, giving uterotonic drugs to enhance contraction of uterine muscles, carrying out controlled cord traction and carrying out uterine massage; a little over half of the ANMs had received training in conducting all of these tasks.

\section{Awareness of maternal and newborn care practices is not comprehensive}

Awareness of best maternal and newborn care practices was, for the most part, high among the ANMs. For example, awareness of the number of antenatal check-ups, the appropriate timing of the first antenatal check-up, number of doses of tetanus toxoid injection, appropriate timing for receiving the first dose of TT injection and number of days that iron and folic acid supplements should be taken was almost universal. However, awareness of the appropriate timing of the second and third checkups and the number of days that a severely anaemic woman should take iron and folic supplements was somewhat limited.

The majority of the ANMs were aware of some of the essential birth preparations; however, only $44 \%$ were able to spontaneously mention three or more preparations for a safe delivery. Awareness of standard protocols to be followed during labour and delivery was limited; only $30 \%$ of ANMs knew that it is not advisable to conduct a vaginal examination if the woman is bleeding at the time of labour or to give oxytocin to speed up delivery, and that using oxytocin or misoprostol for active management of the third stage of labour is recommended.

Awareness of postpartum care practices among the ANMs was far from universal. Only four-fifths of the ANMs knew that a woman should have her first check-up within a few hours or at best within two days of delivery. Only half of the ANMs knew that a woman should have three postpartum check-ups.

Findings show that the vast majority of the ANMs were aware of best practices related to the immediate care of the newborn. Awareness of appropriate feeding practices and the recommended immunization schedule, likewise, was high.

The majority of the ANMs were aware of complications during pregnancy and complications among the newborns; three-quarters were able to list at least three severe complications during pregnancy 
and a little over half were able to list at least three severe complications in the newborns. However, fewer were aware of complications during labour and delivery and the postpartum period; just 32\% and $19 \%$ were able to list at least three severe complications during labour and delivery, and the postpartum period. Findings, moreover, indicate limited awareness of appropriate health facilities for treating pregnancy-related complications. For example, just one-third of the ANMs knew that a pregnant woman who experiences puffiness of the face must go to a primary health centre and a woman who experiences labour lasting more than 12 hours should go to a first referral unit, a community health centre.

Finally, the ANMs' awareness of proper management of selected complications was limited. For example, only $16 \%$ of ANMs knew that if a pregnant woman who experiences pre-eclampsia or whose delivery is imminent approaches the ANM, she should conduct the delivery rather than refer the patient to an FRU without conducting the delivery.

\section{Standard protocols of care are not often followed while delivering maternal and newborn care-related services}

All the ANMs who participated in the study reported that they had provided maternal and newborn care-related services in the three months preceding the interview. Notably, some $37 \%$ of ANMs had conducted a delivery in the three months preceding the interview. However, standard protocols of care were not often followed while delivering these services and the services that were extended were far from comprehensive.

In the area of antenatal care services, findings show that while almost all the ANMs reported that they took a medical history whenever a pregnant woman came for her first antenatal check-up, it was largely limited to enquiring about the woman's previous pregnancy history. Likewise, although over $90 \%$ of ANMs reported that they conducted physical examinations as part of antenatal checkups, these comprised just measuring weight and, to some extent, conducting an abdominal examination and checking blood pressure. No more than half of the ANMs reported that they had conducted basic laboratory investigations. While almost all the ANMs reported that they spent some 10-15 minutes in consultation and that they always asked the woman whether she had any questions to discuss with them, auditory privacy was ensured by just a few and even visual privacy was not ensured by all.

With regard to delivery care, findings show that most of the ANMs who had conducted a delivery in the three months prior to the interview reported that they followed several of the standard protocols - conducting all the essential examinations whenever a woman was admitted to the facility for delivery, using clean gloves while conducting vaginal examinations and washing hands before and after conducting vaginal examinations. However, just a few ANMs reported following such protocols as conducting vaginal examinations about once every four hours and not more often, using the partograph and using oxytocin or misoprostol for the active management of the third stage of labour. Findings also indicate that such harmful practices as applying strong fundal pressure before the woman delivered and using intra-muscular injection to speed up delivery were common, particularly the latter. All the ANMs reported that they ensured visual privacy for the women during delivery and the majority reported that they discharged the women only after at least 24 hours of delivery. However, most ANMs reported that they asked the women or their family members to arrange medicine and other supplies, 
and few allowed the person accompanying the women to remain with them during delivery.

Almost all the ANMs reported that they had visited recently delivered women in the last three months, although not many made the first postpartum visit within 2 days of delivery. The majority of the ANMs reported that they usually counseled recently delivered women about selfcare, care of the newborn, breastfeeding and contraception. Finally, almost all the ANMs reported that they had followed best practices related to newborn care, for example, never delivering the newborn on to the floor, cutting the cord after 2-3 minutes of delivery, not applying any substance on the cord stump, always wiping the newborn dry and wrapping immediately instead of bathing her/him, encouraging the mother to breastfeed the newborn immediately and encouraging the mother to feed colostrum to the newborn.

\section{Awareness of the JSY among ANMs is far from comprehensive}

Awareness of the JSY programme among ANMs was far from comprehensive. The vast majority of the ANMs were aware of most of the eligibility conditions for availing of JSY benefits, including the fact that women could access JSY benefits regardless of their age, parity and household economic status, as well as the type of government health facility in which they could deliver to obtain these benefits. However, their awareness of the monetary and non-monetary support to which women are entitled under the JSY was somewhat limited. For example, only 81 percent of the ANMs knew that a woman in a rural area is entitled to Rs. 1,400 and that a woman in an urban area is entitled to Rs. 1,000 if she delivers in a government or accredited private facility. Findings, moreover, indicate considerable confusion among the ANMs with regard to the entitlements of ASHAs who assist women in accessing JSY benefits.

\section{Recommendations}

The findings of the study suggest a number of priority areas for action.

Increase investments in training programmes for ANMs and raise awareness of the lesser known best practices related to maternal and newborn care and complications during delivery and the postpartum period and management of these complications

Findings that not all ANMs had received the full training in providing maternal and newborn health services call for increased investments in training programmes for ANMs. Findings call for further efforts to raise ANMs' awareness of maternal and newborn care practices. These efforts must pay special attention to increasing their awareness of the complications that a woman may experience during labour and delivery, and the postpartum period, and the appropriate health facilities for treating pregnancy-related complications. Such efforts must also impart information to the ANMs about how to manage complications that ANMs are expected to take care of.

\section{Encourage ANMs to provide delivery and postpartum care-related services on a more regular basis}

Our findings indicate that antenatal care-related services were provided by almost all the ANMs. However, just one third had provided delivery care, and very few had made postpartum visits to recently delivered women within two days of delivery. These findings call for emphasising the role of ANMs 
in providing delivery and postpartum care-related services.

\section{Make special efforts to enable ANMs to follow standard protocols of care}

Findings emphasise the need for special efforts to enable ANMs to follow standard protocols of care. Specifically, ANMs need to be oriented about the importance, while taking a woman's medical history, of enquiring about the date of the last menstrual period, any history of systemic illnesses, and symptoms experienced during pregnancy as well as checking blood pressure, pallor and height while conducting antenatal check-ups. Likewise, in the area of delivery care, ANMs need to be trained and encouraged to use the partograph for monitoring labour and to use oxytocin or misoprostol for the active management of the third stage of labour. At the same time, they must be dissuaded from such harmful practices as applying fundal pressure before the woman delivers and using intra-muscular injections to speed up delivery. In the area of postpartum care, our findings call for orienting ANMs about the importance of making the first postpartum visit to recently delivered women within two days of delivery. Enabling ANMs to follow these standard protocols of care may require training programmes to upgrade their skills. The training must emphasise the need to provide good quality services as much as it focuses on developing technical skills. Also required are monitoring mechanisms to ensure that ANMs do follow the protocols.

We also note that improvements in infrastructure at the health facility are required to enable ANMs to follow standard protocols of care, for example, to ensure privacy for the women or keep the delivered women at the facility for more than a day.

\section{Raise ANMs' awareness about the JSY} programme and address weaknesses in the programme identified by $A N M s$

ANMs recognised that the JSY had resulted in an increased demand for maternal health services. However, they were not fully aware of women's entitlements; efforts are needed to provide comprehensive information to ANMs about the JSY programme and its entitlements. At the same time, efforts must be made to address issues raised by ANMs about weaknesses in the implementation of the programme, for example, about inadequate staff at the PHC, lack of facilities for conducting deliveries at the sub-centre and JSY payment linked only to institutional delivery rather than a continuum of care, including immunization of infants. 


\section{References}

Academy for Nursing Studies and Women's Empowerment Research Studies (ANSWERS) and National Health System Resource Centre (NHSRC). 2010a. Study Report on Nursing Services in Bihar: Current Situation, Requirements and Measures to Address Shortages. Hyderabad: ANSWERS.

Academy for Nursing Studies and Women's Empowerment Research Studies (ANSWERS) and National Health System Resource Centre (NHSRC). 2010b. Study Report on Nursing Services in Chhattisgarh: Current Situation, Requirements and Measures to Address Shortages. Hyderabad: ANSWERS.

Academy for Nursing Studies and Women's Empowerment Research Studies (ANSWERS) and National Health System Resource Centre (NHSRC). 2010c. Study Report on Nursing Services in Orissa: Current Situation, Requirements and Measures to Address Shortages. Hyderabad: ANSWERS.

Academy for Nursing Studies and Women's Empowerment Research Studies (ANSWERS) and National Health System Resource Centre (NHSRC). 2010d. Study Report on Nursing Services in Rajasthan: Current Situation, Requirements and Measures to Address Shortages. Hyderabad: ANSWERS.

Academy for Nursing Studies and Women's Empowerment Research Studies (ANSWERS) and National Health System Resource Centre (NHSRC). 2010e. Study Report on Nursing Services in Uttarakhand: Current Situation, Requirements and Measures to Address Shortages. Hyderabad: ANSWERS.

Bhatia, J.C. 1999. Constraints to the quality of primary health services in rural Karnataka, in M.A. Koenig and M.E. Khan (eds.) Improving Quality of Care in India's Family Welfare Programme: The Challenge Ahead, New Delhi: Population Council, pp. 182-209.

IntraHealth. 2008. Skilled Birth Attendance by Auxiliary Nurse Midwives in Select Districts of Jharkhand. Technical report, Vistaar Project.

International Institute for Population Sciences (IIPS) and Macro International. 2008. National Family Health Survey (NFHS-3), India, 2005-06: Rajasthan. Mumbai: IIPS.

International Institute for Population Sciences (IIPS). 2010. District Level Household and Facility Survey (DLHS-3), 2007-08: India. Mumbai: IIPS.

Iyer, A and A. Jesani. 1999. Barriers to the quality of care: the experience of auxiliary-nurse-midwives in rural Maharashtra, in M.A. Koenig and M.E. Khan (eds.) Improving Quality of Care in India's Family Welfare Programme: The Challenge Ahead, New Delhi: Population Council, pp. 210-237.

Khan, M.E., B.C. Patel and R.B. Gupta. 1999. The quality of family planning services in Uttar Pradesh from the perspectives of service providers, in M.A. Koenig and M.E. Khan (eds.) Improving Quality of Care in India's Family Welfare Programme: The Challenge Ahead, New Delhi: Population Council, pp. 238-269.

Mavalankar, D. and K.S. Vora. 2008. The Changing Role of Auxiliary Nurse Midwife (ANM) in India: Implications for Maternal and Child Health (MCH). Ahmedabad: Indian Institute of Management.

Mavalankar, D., K.S. Vora and M. Prakasamma. 2008. Achieving millennium development goal 5: Is India serious? Bulletin of the World Health Organization, 86(4): 243-4. 
Ministry of Health and Family Welfare (MOHFW). 2005. Guidelines for Antenatal Care and Skilled Attendance at Birth by ANMs and LHVs. New Delhi: Maternal Health Division, Government of India.

Ministry of Health and Family Welfare (MOHFW). 2006a. Indian Public Health Standards (IPHS) for Sub-centres Guidelines. New Delhi: Directorate General of Health Services, Government of India.

Ministry of Health and Family Welfare (MOHFW). 2006b. Facilitator's Guide for Conducting Training for ANMs, LHVs and Staff Nurses as Skilled Birth Attendant, New Delhi: Maternal Health Division, Government of India.

Ministry of Health and Family Welfare (MOHFW). 2010. Guidelines for Antenatal Care and Skilled Attendance at Birth by $A N M s / L H V_{s} / S N s$. New Delhi: Maternal Health Division, Government of India.

Ministry of Health and Family Welfare (MOHFW). 2011. Rural Health Statistics in India 2010. New Delhi: MOHFW, Government of India. Accessed on 21 December 2011 at http://nrhm-mis.nic.in/UI/RHS/ RHS\%202010/Rural\%20Health\%20Statistics\%202010.htm.

Office of the Registrar General, India. 2011. Special bulletin on maternal mortality in India 2007-09, Sample Registration System. New Delhi: Office of the Registrar General, India.

Office of the Registrar General and Census Commissioner, India. 2011. Provisional Population Totals, Paper I of 2011: Census of India 2011, Series I-India. New Delhi: Office of the Registrar General, India.

Santhya, K.G., S.J. Jejeebhoy and A.J. Francis Zavier. 2011. Implementing the Janani Suraksha Yojana: Perspectives and experiences of Accredited Social Health Activists in Rajasthan. New Delhi: Population Council.

United Nations Population Fund (UNFPA). 2009. Concurrent Assessment of Janani Suraksha Yojana (JSY) Scheme in Selected States of India, 2008. Accessed on 22 September, 2010 at http://www.mohfw.nic.in/NRHM/ Documents/JSY_Study_UNFPA.pdf.

United Nations Children Fund (UNICEF). 2010. Coverage Evaluation Survey 2009. UNICEF: New Delhi.

Ved, R.R. and A.S. Dua. 2005. Review of women and children's health in India: Focus on safe motherhood, in National Commission on Macroeconomics and Health (ed.) Background Papers, Burden of Disease in India, New Delhi: National Commission on Macroeconomics and Health.

Verma, R.K. and T.K. Roy. 1999. Assessing the quality of family planning service providers in four Indian states, in M.A. Koenig and M.E. Khan (eds.) Improving Quality of Care in India's Family Welfare Programme: The Challenge Ahead, New Delhi: Population Council, pp. 169-182.

Visaria, L. 1999. The quality of reproductive health care in Gujarat: perspectives of female health workers and their clients, in M.A. Koenig and M.E. Khan (eds.) Improving Quality of Care in India's Family Welfare Programme: The Challenge Ahead, New Delhi: Population Council, pp. 143-168.

Vora, K.S., D. Mavalankar, K.V. Ramani et al. 2009. "Maternal health situation in India: A case study," Journal of Health, Population and Nutrition, 27(2): 184-201. 


\section{Members of the study team}

$\begin{array}{ll}\text { Investigators } & \\ \text { Babita Bansod } & \text { Neha Panday } \\ \text { Geeta Gupta } & \text { Prem Kanwar Rathore } \\ \text { Priya Gupta } & \text { Premlata Rathore } \\ \text { Ravi Jain } & \text { B.Srihari } \\ \text { Binit Jha } & \text { Rituraj Solanki } \\ \text { Dlilshad Khan } & \text { Mukesh Vijay } \\ \text { Najma Khan } & \text { Pushplata Yadav } \\ \text { Nikhat Khan } & \text { Radha Yadav } \\ \text { Manisha Kaushal } & \text { Rukmani Yadav } \\ \text { Irshad Mohammad } & \text { Sheela Yadav }\end{array}$

Editor

Shanti Menon

Komal Saxena

Administration

M A Jose 


\section{Authors}

K G Santhya, Associate II, Population Council, New Delhi

Shireen J Jejeebhoy, Senior Associate, Population Council, New Delhi 


\section{Population Council}

Zone 5-A, Ground Floor

India Habitat Centre

Lodi Road

New Delhi 110 003, India 